\title{
Periodic Space Partitioners (PSP) and their relations to Crystal Chemistry
}

\section{Journal Article}

\section{Author(s):}

Nesper, Reinhard; Grin, Yuri

Publication date:

2011-08

Permanent link:

https://doi.org/10.3929/ethz-b-000041261

Rights / license:

In Copyright - Non-Commercial Use Permitted

Originally published in:

Zeitschrift für Kristallographie 226(8), https://doi.org/10.1524/zkri.2011.1429 


\title{
Periodic Space Partitioners (PSP) and their relations to crystal chemistry
}

\author{
Reinhard Nesper and Yuri Grin* \\ In memoriam Professor Dr. Dr. h.c. mult. Hans Georg von Schnering
}

Received June 27, 2011; accepted July 7, 2011

\begin{abstract}
We are looking back on 30 years development of periodic space partitioners (PSP) and their relations to their periodic relatives, i.e. minimal surfaces (PMS), zero potential surfaces (POPS), nodal surfaces (PNS), and exponential scale surfaces. Hans-Georg von Schnering and Sten Andersson have pioneered this field especially in terms of applications to crystal chemistry. This review relates the early attempts to approximate periodic minimal surfaces which established a systematic classification of all PSP in terms space group symmetry and consecutive applications in a variety of different fields. A consistent nomenclature is outlined and different methods for deriving PSP are described. Characteristic structure factor sets which solely define PNS by can be used to discriminate structure types of a given symmetry or even to determine complicated crystal structures. The concept of PSP relates space group symmetry, topology, and chemical bonding in an intriguing way and tessellations on PSP which can be generated in a straight forward way allow to predict new framework types. Through transformation of such continuous topological forms a new entry has been found for understanding and interpreting reconstructive phase transitions. Finally we indicate the importance of PSP models for soft matter science.
\end{abstract}

\section{History and development}

Minimal surfaces are a middle aged problem in mathematics mostly addressed since the $19^{\text {th }}$ century, but constitute an eternal fascination for humans probably since the earliest days of conscious thought. The intriguing fascination of soap bubbles is related to a deep feeling of harmony and beauty, the priming of which we simply do not know [1]. It may be due to a comprehensive intuitive understanding of the world without concrete knowing just like the feeling of harmony which is created by the golden ratio and five-fold symmetry. Why does the most irrational number ${ }^{l}$ induce such a state of comfort in human minds? We do not know, just as nobody knows why the fine struc-

\footnotetext{
* Correspondence author (e-mail: grin@cpfs.mpg.de)

1 Every irrational number can be represented by a continued fraction; for the golden ratio only the number one occurs in this series which makes it the slowest converging continued fraction.
}

ture constant approaches more and more the value 1/137, the denominator being $33^{\text {rd }}$ prime number. To solve this question was the last great goal of Wolfgang Pauli who was not only a great physicist and mathematician but also stressed the importance of intuitive solutions to physical problems, amongst others by dreaming [2].

A mathematical treatment of minimal surfaces goes back to Lagrange's work in the $18^{\text {th }}$ century which resulted in the Euler-Lagrange equation [3]. In the $19^{\text {th }}$ century the experiments of $\mathrm{J}$. Plateau contributed much to mathematical research on such objects and led to the formulations of Plateau's problem which turned out to be unsolvable in general analytical form. The quest for deriving minimal surfaces and periodic minimal surfaces as well as for their analytical formulation increased by the end of the $19^{\text {th }}$ century especially by H. A. Schwarz [4] and in the beginning of the $20^{\text {th }}$ century by D. Hilbert [5]. Hilbert was not only one of the greatest mathematicians of modern age but also a mathematical purist who outlawed experiments for mathematicians. Despite this he exempted one, namely the soap bubble experiment because of the difficulties with Plateau's problem [6].

By the midst of the $20^{\text {th }}$ century some institutions explored possible applications for Periodic Minimal Surfaces (PMS) like the American space agency NASA under the leadership of the mathematician H. A. Schoen [7]. Schoen derived the so-called Gyroid PMS which belongs to the highest 3D space group no 230 but misassigned it to the direct subgroup no. 214. In his report he poses with a model of the surface but he does not look very happy, presumably because the difficulties of treating such a problem in a mathematically rigorous way. The interest of researchers and engineers in PMS is straightforward because one of their characteristics is to span a 3D frame by the least surface area possible, that is the by least amount of material, by the lowest weight, and eventually by the highest specific strength, etc. In addition, it seemed likely that such forms constitute most stable construction schemes because of their optimal form. Seemingly that project was given up or postponed by NASA because of enormous obstacles for useful realization at that time.

Simultaneously, artists explored that field. A number of architects and artists began to work with local and periodic minimal surfaces in the 1950ies (Fig. 1). F. Otto started to develop light-weighted constructions by help of soap bub- 
Fig. 1. Examples from architecture and from arts for utilization of minimal surfaces: (left) Michael Burt [11]; (upper - right) - Frei Otto, soap bubble model; (lower - right) - Frei Otto, Tanzbrunnen Köln [12].

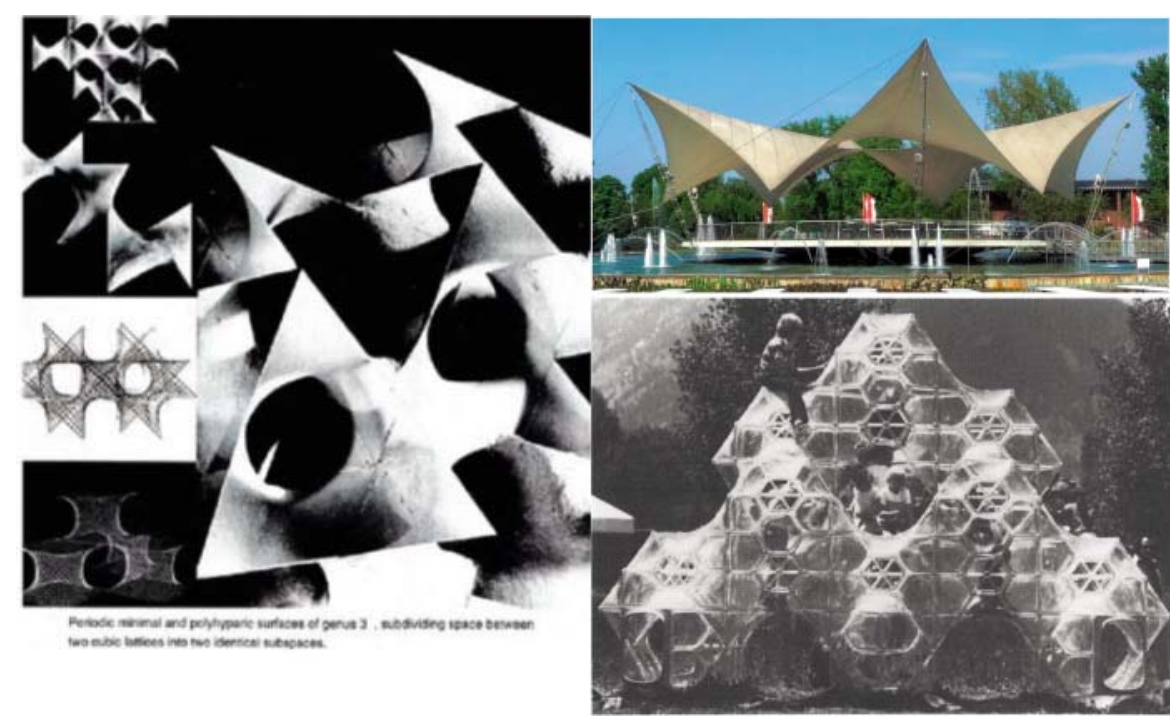

ble experiments [8]. Another architect, Michael Burt, developed extensively models and based his constructions to a large extent on minimal surface and especially on Periodic Minimal Surface and Voronoi cell approaches [9, 10].

The artist Angel Duarte deeply explored minimal surface objects for designing a specific kind of sculptures since the 1970ies [13]. He very often constructed an outer frame of a surface patch by a metal frame and then filled in straight metal rods such that they most closely related to the real minimal surface of the boundary frame. In a more design-based approach Peter Pearce utilized minimal surface techniques for creating objects of arts but also of daily use.

Although some objects like Fermi surfaces in physics, like clathrate structures in structural chemistry, and vesicle/micelle in tenside research did exemplify the importance of Minimal Surfaces as well as Periodic Minimal Surfaces as topological representations of quite different phenomena (Fig. 2) it was not until the beginning of the 1980ies that the general meaning of Periodic Minimal Sur-

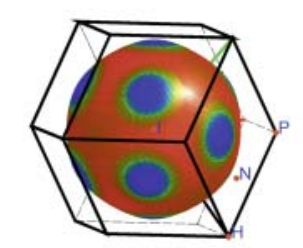

$\mathbf{a}$

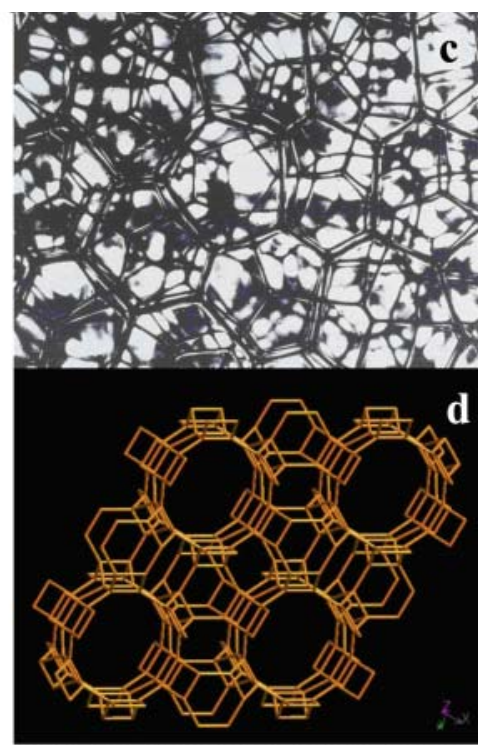

Fig. 2. (a) Fermi surface of copper, (b) Fermi surface of rubidium, (c) framework of soap bubbles, (d) model of the zeolite MAZ) [15]. faces and other Periodic Space Partitioners (PSP) for periodic matter was noted in natural sciences. It should be noted, PSP can be periodic hyperbolic surfaces, but need not be.

It was Sten Andersson, an ingenious structural chemist who initiated a general attempt to correlate crystal structures and PMS with enthusiastic scientific power [14]. This was the starting point of systematic investigations which lead to a general relation of space groups and periodic minimal surfaces and related periodic surfaces.

\section{Homogeneous sphere packings \& continuous networks - atoms on wire Gitterkomplexe - lattice complexes}

The term lattice complex was coined by Paul Niggli and later adopted by Carl Hermann into the International Tables of Crystallography [16]. A more precise specification of the term was given later [17]. Lattice complexes may be developed by one representative point set (Wyckoff set) of a space group [18]. The analysis of all characteristic Wyckoff (which have no free parameter) sets in all space groups results in 25 invariant lattice complexes, 12 invariant lattice complexes for non-characteristic ones and 106 non-invariant lattice complexes [16]. Placing an entity, i.e. point, spin, atom etc. on such Wyckoff site develops into a representation of the lattice complex. A well known example yield the sets $1 a$ and $1 b$ in the space group $P m \overline{3} m$ each of which generate a cubic primitive graph when connected. The two graphs set up isomorphous interpenetrating networks which are shifted by the vector $1 / 21 / 21 / 2$ against each other. The two graphs are merged into one set by the super group $\operatorname{Im} \overline{3} m$ through the set $2 a$ which carries the special lattice complex symbol $I$. Historically this is being used to designate body centering and in the general nomenclature $I=P^{*}$, where the star denotes the shift vector of $1 / 21 / 21 / 2$ of the former subsets. The lattice complex graphs can be located either in the labyrinths of the according to a PNS partitioned space or on that PNS. Some examples of open frameworks and their enveloping surfaces are shown in Fig. 3. 

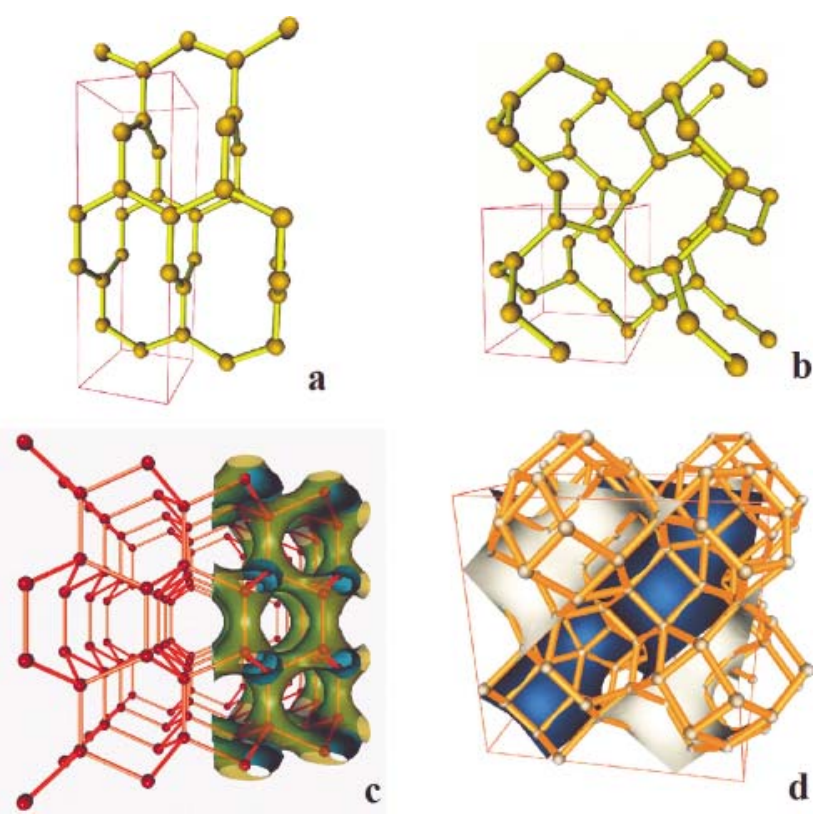

Fig. 3. Some open frameworks (a) silicon net in the $\mathrm{ThSi}_{2}$ structure; (b) silicon net in the $\mathrm{SrSi}_{2}$ structure; (c) diamond net and enveloping surface parallel to the $D$ surface; (d) hypothetical framework following the $G$ surface [19].

If one expands such point sets into their dedicated Wirkungsbereiche [20], Dirichlet [21] domains, or Voronoi [22] polyhedra then a connected structure develops which, in general, has an orientable periodic surface in three dimensions (Fig. 4). Such Periodic Wirkungsbereich Surfaces (PWS) are polygonal approximants to curved hyperbolic surfaces.
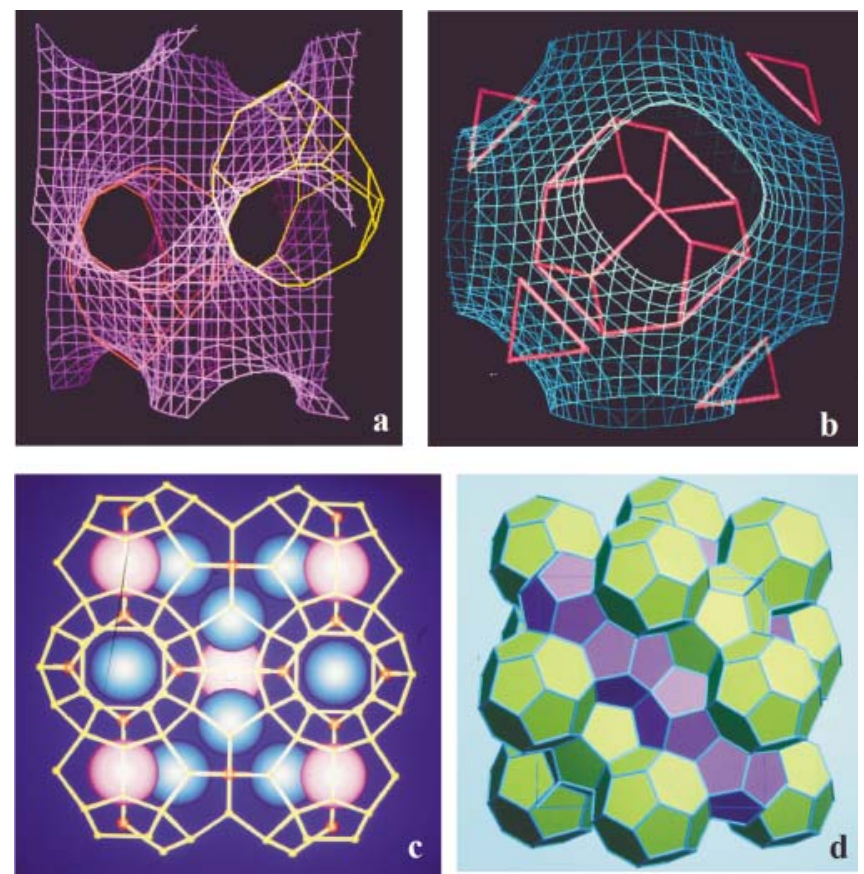

Fig. 4. (a) Dirichlet domains and Gyroid surface in space group $I a \overline{3} d$; (b) Dirichlet domains and $P$ surface in space group $\operatorname{Im} \overline{3} m$; projection of the clathrate I structure visualizing domains around the cation centers (big spheres); (d) polyhedral display of the clathrate II structure.
A quite versatile systematic approach to PWS is based on the so-called circle and sphere packings which originally were derived from the concept of Bauverbände or homogeneous frameworks due to Hellner et al. [23]. In a crystal structure, the Dirichlet domains around certain sites touch most fundamentally (with the largest surface area) along vectors to the closest neighbors. Accordingly, one can construct the space group specific frameworks also through packings of touching spheres of the same size. This is the reason why they have been called homogeneous sphere packings (HSP). In general, these sphere packings are not dense. There are 199 distinct HSP for the cubic crystal system, 394 for tetragonal, 170 for hexagonal and yet unknown numbers for lower symmetry HSPs [24]. Crystallography and structure chemical formalisms in form of general networks reminded very much to PMS. It should be noted that A. F. Wells has given a quite comprehensive survey of $2 \mathrm{D}$ and $3 \mathrm{D}$ nets which are realized in crystal structures, at a time [25]. Another large overview of mostly interpenetrating sets occurring in intermetallic phases is contained in W. B. Pearson's book on intermetallic compounds [26].

\section{Methods of construction}

Due to the enormous difficulties in deriving and calculating new PMS through Gauss mapping and solving Weierstrass equations from scratch it was eligible to develop another approach. Beyond such general obstacles, a clear connection to space group symmetry was still missing until the midst of the 1980ies. The notion that a field approach instead of a surface centered view can lead to similar periodic forms though not necessarily with minimal surface properties opened the way for a direct derivation of periodic hyperbolic surfaces from space groups. The first attempts in this direction utilized Coulombic fields in which periodic equipotential and zero surfaces (P0Ps) were calculated and finally represented. Indeed, the simplest charge distribution in the corresponding symmetry groups $F d \overline{3} \mathrm{~m}$, $P m \overline{3} m$, and $I a \overline{3} d$ did yield the Schwarz $D$, Schwarz $P$, and Schoen's $G$ (gyroid) surfaces. It became immediately clear how to proceed: the POPS corresponding to Schwarz's $P$ surface is calculated from a cesium chloride-kind of charge distribution (Fig. 6a, [27, 107]). While both the plus and the minus potential spaces belong to space group $P m \overline{3} m$, the POPS must have higher symmetry due to the fact that negative and positive spaces are congruent and only shifted by $1 / 21 / 21 / 2$ with respect to each other. A surface having topologically identical spaces on both sides is called a balanced surface. In such a case additional symmetry elements have to be on that surface which exchanges topologically both spaces. These belong to a set of twofold axes which of course do not appear in $P m \overline{3} m$ but in the supergroup $\operatorname{Im} \overline{3} m$ (Wyckhof set $48 i$ ). Twofold axes running along the surface must necessarily be identical with straight lines on the surface. These straight lines set up on the one hand the wireframes for the minimal surface soap skin experiment, on the other they delimit the smallest asymmetric unit of the unit cell which, through rotations, inversions, and mirroring, develops into the complete surface. The symbolic 
description for generating such a surface was formulated initially as POPS:

Schwarz $P$ or $P^{*} \quad P_{(1 a)}^{(+)} \wedge P_{(1 b)}^{(-)}$or $\operatorname{cl}\left(P I^{1 / 2} 1 / 21 / 2 I P\right)$

by referring to the lattice complex notation. It says that two primitive $(P)$ lattice complexes which are related by a body centering carry the charges given in the superscript $(+1)$ and $(-1)$ and are located in the Wyckoff sites given as subscripts. The short symbol for such a body centered combination is the *.

In an attempt to relate the coding of different PS and to allow better comparison we rewrite to

$$
\begin{array}{ll}
\text { POPS: Schwarz } P \text { or } P^{*} \quad & P m \overline{3} m<(000)^{+1} ; \\
& (1 / 21 / 21 / 2)^{-1}>\operatorname{Im} \overline{3} m
\end{array}
$$

starting with the generating space group symmetry, followed by brackets \langle\rangle and ending with the resulting POPS space group symmetry. The brackets \langle\rangle contain the information about the location $(x, y, z)$ of the charge and its magnitude and sign as an superscript [28]. In general, from

$$
\text { POPS: } \quad \mathbf{H}<\left(x_{+}, y_{+}, z_{+}\right)^{+1} ;\left(x_{-}, y_{-}, z_{-}\right)^{-1}>\mathbf{G}
$$

it is clear that the additional symmetry elements of $\mathbf{G}$ with respect to it's subgroup $\mathbf{H}$ must be part of the POPS and must be invariant parts of the POPS [27]. This led already in the beginning to an instantaneous classification of 24 P0PS and related PMS many of which were new and not yet known at that time.

At this point it should be noted that balanced surfaces establish black and white groups which are appropriate descriptors for physical situations like antiferromagnetic spin structures. Clearly, this insight right in the midst of the 1980ties paved the way for a systematic derivation of balanced periodic surfaces through group-subgroup relations [16] which was the necessary prerequisite [29] for the later systematic work of Fischer and Koch [30]. Consecutively a primary table of P0Ps was derived from systematic analysis of black and white symmetry groups [31].

It is also an interesting, yet unanswered question, how three dimensional $n$-periodic surfaces develop in $n$-color groups, i.e. triply periodic surfaces in three-color groups. Fogden and Hyde give an example of a balanced triply periodic surface on their homepage [31] while Holyst simply derives tripling of space by generating parallel surfaces. This, of course, can be done in an infinite manner and only emphasizes the necessary laminate-like nature of periodic 2D surfaces filling 3D space [32].

Since P. P. Ewald's centennial work on lattice sums it was clear that Coulombic interactions can be represented both in real (by the summation over the Coulomb formula) and in reciprocal space (by summation over structure factors which are derived from a charge distribution). While the real space sum does not converge, the reciprocal does quickly. Felix Bertaut proposed in the 1950ies to completely represent the periodic Coulomb summation by a short Fourier series [33] and he was able to show that just a few principle structure factors close to the origin, i.e. with smallest $\mathbf{k}$-vectors can cope for sufficiently precise calculation of Madelung constants and Madelung parts of lattice energy (MAPLE). It is also interesting to note, that Bertaut published a formula for MAPLE which consists of two terms: a structure dependent term which consists of a few principal structure factors or most fundamental Bragg reflections, and a structure independent term only taking into account the existence of discrete charges $q_{i}$ at a mean distance $R$ (negative term on the right):

$$
U=\lambda \sum_{h} \frac{F_{h}^{2} \varphi_{h}^{2}}{h^{2}}-\frac{3}{5} \cdot \frac{1}{R} \sum_{i} q_{i}^{2} .
$$

The first term adds concrete topological information through a short series of charge structure factors which are defined according to [34]

$$
F(\mathbf{h})=V \int_{0}^{1} \rho(r) \mathrm{e}^{2 \pi i(\mathbf{h} r)} \mathrm{d} r .
$$

The charge density function $F(\mathbf{h})$ consists only of the structure dependent part.

$$
\rho(x)=\frac{1}{V} \int_{-\infty}^{+\infty} F(\mathbf{h}) \mathrm{e}^{-2 \pi i(\mathbf{h} x)} \mathrm{d} h .
$$

By the end of the 1980ies there was a correspondence with Bertaut through whether or not only one structure factor set representing properly the space group in question was simply a representative of symmetry, free of physical meaning or still a representation of Coulombic fields. Well, it turned out that it is both, dependent on which glasses are worn [35]. The generation of Schwarz $P$ surface by the set of reciprocal vectors $\{100\}$ with amplitude 1.0 and phase angle $\alpha=0$ (consisting of the vectors 100 , 001 , and 010 to properly represent cubic symmetry) does not contain any physical information and thus can only generate a spatial representation of $P m \overline{3} m$ symmetry where the trace of density $\rho=0$ belongs to both negative and positive density and thus enters into the supergroup $\operatorname{Im} \overline{3} m$. However, if one assigns a reciprocal charge unit to the amplitude then a representation of the Coulombic field results which, of course is topologically completely identical to the aforementioned unphysical representation. So, just like cubic harmonic functions being on the one hand functions of electron orbitals of the atoms, they are on the other hand, in a much more general sense, representations of the group of the sphere (= point group K) without any specific physical meaning. The first large set of PNS was published $1990[36,37]$ and is given in the Table 1.

\section{Definitions}

In the following we shall use the PNS calculated as

$$
R(\vec{r})=\sum_{n=1}^{N}\left|S\left(\mathbf{h}_{n}\right)\right| \underset{n}{k} \cos \left(2 \pi \mathbf{h}_{n} \mathbf{r}-\alpha\left(\mathbf{h}_{n}\right)\right)=0
$$

Where $S\left(\mathbf{h}_{n}\right)=\sum_{i} \delta_{i} \exp \left(2 \pi i \mathbf{h}_{n} \mathbf{r}_{i}\right)$ is the geometric structure factor; $\delta_{\mathrm{i}}$ stands for a dimensionless $\delta$-function, $k_{n}=\left(\frac{\left|\Psi_{n}\right|}{|\mathbf{h}|}\right)^{2}$ is a decay function; $\mathbf{h}$ and $\mathbf{r}$ are the vectors in reciprocal and direct space, $\mathbf{h}=h \mathbf{a}^{*}+k \mathbf{b}^{*}+l \mathbf{c}^{*}$ (in 


\begin{tabular}{|c|c|c|c|c|c|c|c|c|}
\hline $\begin{array}{l}\text { Symbol } \\
\text { after [24] }\end{array}$ & $\begin{array}{l}\text { Symbol } \\
\text { after }[24]\end{array}$ & $h k l$ & $|S| \times w$ & $\alpha$ & $\begin{array}{l}\text { Generating space } \\
\text { group }\end{array}$ & $f(x, y, z)=0$ & $\begin{array}{l}\text { Symmetry } \\
\text { of surface }\end{array}$ & Symbol after [28] \\
\hline$P^{*}$ & $P$ & 100 & 1 & 0 & $P m \overline{3} n$ & $\cos X+\cos Y+\cos Z=0$ & $\operatorname{Im} \overline{3} m$ & $P m \overline{3} m<(100)_{0}^{1}>\operatorname{Im} \overline{3} m$ \\
\hline$F^{*}$ & & 111 & 1 & 0 & $F m \overline{3} m$ & $\cos X \cdot \cos Y \cdot \cos Z=0$ & $P m \overline{3} m$ & $F m \overline{3} m<(111)_{0}^{1}>P m \overline{3} m$ \\
\hline$D^{*}$ & $D$ & 111 & 1 & $\begin{array}{l}0 \\
-\pi / 4\end{array}$ & $\begin{array}{l}F d \overline{3} m \\
\text { (origin at }-3 \mathrm{~m} \text { ) } \\
F d \overline{3} m \\
\text { (origin at }-43 \mathrm{~m} \text { ) }\end{array}$ & $\begin{array}{l}\cos (X-Y) \cdot \cos Z+\sin (X+Y) \cdot \sin Z=0 \\
\cos X \cdot \cos Y \cdot \cos Z+\sin X \cdot \sin Y \cdot \sin Z=0\end{array}$ & $\operatorname{Pn} \overline{3} m$ & $\begin{array}{l}F d \overline{3} m<(111)_{0}^{1}>P n \overline{3} m \\
F d \overline{3} m<(111)_{-\pi / 4}^{1}>P n \overline{3} m\end{array}$ \\
\hline$Y^{* *}$ & $Y^{*}, G$ & 110 & 1 & $\pi / 2$ & $I 4_{1} 32$ & $\sin X \cdot \cos Y+\sin Y \cdot \cos Z+\cos X \cdot \sin Z=0$ & $I a \overline{3} d$ & $I 4_{1} 32<(110)_{\pi / 2}^{1}>I a \overline{3} d$ \\
\hline$I P_{2}-J^{*}$ & $I-W P$ & $\begin{array}{l}110 \\
200\end{array}$ & $\begin{array}{l}1 \\
1\end{array}$ & $\begin{array}{l}0 \\
\pi\end{array}$ & $\operatorname{Im} \overline{3} m$ & $2 \cdot[\cos X \cdot \cos Y+\cos Y \cdot \cos Z+\cos X \cdot \cos Z]-[\cos 2 X+\cos 2 Y+\cos 2 Z]=0$ & $\operatorname{Im} \overline{3} m$ & $\operatorname{Im} \overline{3} m<(110)_{0}^{1}(200)_{\pi}^{1}>\operatorname{Im} \overline{3} m$ \\
\hline$S^{*}$ & $S$ & 211 & 1 & $\pi / 2$ & $I-43 d$ & $\cos 2 X \cdot \sin Y \cdot \cos Z+\cos X \cdot \cos 2 Y \cdot \sin Z+\sin X \cdot \cos Y \cdot \cos 2 Z=0$ & $I a \overline{3} d$ & $I \overline{4} 3 d<(112)_{\pi / 2}^{1}>I a \overline{3} d$ \\
\hline$C\left(D^{*}\right)$ & $C(D)$ & 311 & 1 & $\begin{array}{l}0 \\
-\pi / 4\end{array}$ & $\begin{array}{l}F d \overline{3} m \\
\text { (origin at }-3 \mathrm{~m} \text { ) } \\
F d \overline{3} m \\
\text { (origin at }-43 \mathrm{~m} \text { ) }\end{array}$ & $\begin{array}{l}\cos (3 X+Y) \cdot \cos Z-\sin (3 X-Y) \cdot \sin Z+\cos (X+3 Y) \cdot \cos Z+\sin (X-3 Y) \cdot \sin Z \\
+\cos (X-Y) \cdot \cos 3 Z-\sin (X+Y) \cdot \sin 3 Z=0 \\
\cos 3 X \cdot \cos Y \cdot \cos Z-\sin 3 X \cdot \sin Y \cdot \sin Z+\cos X \cdot \cos 3 Y \cdot \cos Z \\
-\sin 3 X \cdot \sin Y \cdot \sin Z+\cos X \cdot \cos Y \cdot \cos 3 Z-\sin X \cdot \sin Y-\sin 3 Z=0\end{array}$ & $\operatorname{Pn} \overline{3} m$ & $\begin{array}{l}F d \overline{3} m<(113)_{0}^{1}>P n \overline{3} m \\
F d \overline{3} m<(113)_{-\pi / 4}^{1}>P n \overline{3} m\end{array}$ \\
\hline$P^{*} J^{*}$ & $C(P)$ & $\begin{array}{l}100 \\
111\end{array}$ & 1 & $\begin{array}{l}0 \\
0\end{array}$ & $\operatorname{Pm} \overline{3} m$ & $\cos X+\cos Y+\cos Z+4 \cdot \cos X \cdot \cos Y \cdot \cos Z=0$ & $\operatorname{Im} \overline{3} m$ & $\operatorname{Pm} \overline{3} m<(100)_{0}^{1}(111)_{0}^{1}>\operatorname{Im} \overline{3} m$ \\
\hline$C\left(Y^{* *}\right)$ & & $\begin{array}{l}110 \\
310\end{array}$ & $\begin{array}{l}3 \\
2\end{array}$ & $\pi / 2$ & $I 4_{1} 32$ & $\begin{array}{l}3 \cdot[\sin X \cdot \cos Y+\sin Y \cdot \cos Z+\cos X \cdot \sin Z]+2 \cdot[\sin 3 X-\cos Y+\cos X \cdot \sin 3 Z \\
+\sin 3 Y \cdot \cos Z-\sin X \cdot \cos 3 Y-\cos 3 X \cdot \sin Z-\sin Y \cdot \cos 3 Z]=0\end{array}$ & $I a \overline{3} d$ & $I 4_{1} 32<(110)_{\pi / 2}^{3}(310)_{\pi / 2}^{2}>I a \overline{3} d$ \\
\hline$C\left(S^{*}\right)$ & $C(S)$ & $\begin{array}{l}200 \\
321 \\
231\end{array}$ & $\begin{array}{l}2 \\
1 \\
1\end{array}$ & $\begin{array}{l}0 \\
\pi \\
\pi\end{array}$ & $I a \overline{3}$ & $\begin{array}{l}\cos 2 X+\cos 2 Y+\cos 2 Z+2 \cdot[\sin 3 X \cdot \sin 2 Y \cdot \cos Z+\cos X \cdot \sin 3 Y \cdot \sin 2 Z \\
+\sin 2 X \cdot \cos Y \cdot \sin 3 Z]+2 \cdot[\sin 2 X \cdot \cos 3 Y \cdot \sin Z+\sin X \cdot \sin 2 Y \cdot \cos 3 Z \\
+\cos 3 X \cdot \sin Y \cdot \sin 2 Z]=0\end{array}$ & $I a \overline{3} d$ & $\operatorname{Ia} \overline{3}<(200)_{0}^{2}(321)_{\pi}^{1}(231)_{\pi}^{1}>\operatorname{Ia} \overline{3} d$ \\
\hline$I_{2}-Y^{* *}$ & & $\begin{array}{l}211 \\
220\end{array}$ & 1 & $\begin{array}{l}0 \\
0\end{array}$ & $I a \overline{3} d$ & $\begin{array}{l}-2 \cdot[\sin 2 X \cdot \cos Y \cdot \sin Z+\sin X \cdot \sin 2 Y \cdot \cos Z+\cos X \cdot \sin Y \cdot \sin 2 Z] \\
+\cos 2 X \cdot \cos 2 Y+\cos 2 Y \cdot \cos 2 Z+\cos 2 X \cdot \cos 2 Z=0\end{array}$ & $I a \overline{3} d$ & $\operatorname{Ia} \overline{3} d<(211)_{0}^{1}(220)_{0}^{1}>\operatorname{Ia} \overline{3} d$ \\
\hline$C\left(I_{2}-Y^{* *}\right)$ & & $\begin{array}{l}211 \\
220\end{array}$ & $\begin{array}{l}1 \\
1\end{array}$ & $\begin{array}{l}\pi \\
0\end{array}$ & $I a \overline{3} d$ & $\begin{array}{l}+2 \cdot[\sin 2 X \cdot \cos Y \cdot \sin Z+\sin X \cdot \sin 2 Y \cdot \cos Z+\cos X \cdot \sin Y \cdot \sin 2 Z] \\
+\cos 2 X \cdot \cos 2 Y+\cos 2 Y \cdot \cos 2 Z+\cos 2 X \cdot \cos 2 Z=0\end{array}$ & $I a \overline{3} d$ & $\operatorname{Ia} \overline{3} d<(211)_{\pi}^{1}(220)_{0}^{1}>\operatorname{Ia} \overline{3} d$ \\
\hline$W^{*}$ & & 210 & 1 & 0 & $\operatorname{Pm} \overline{3} n$ & $\begin{array}{l}\cos 2 X \cdot \cos Y+\cos 2 Y \cdot \cos Z+\cos X \cdot \cos 2 Z-[\cos X \cdot \cos 2 Y+\cos Y \cdot \cos 2 Z \\
+\cos 2 X \cdot \cos Z]=0\end{array}$ & $\operatorname{Im} \overline{3} m$ & $\operatorname{Pm} \overline{3} n<(210)_{0}^{1}>\operatorname{Im} \overline{3} m$ \\
\hline$Y^{*}$ & Y & $\begin{array}{l}111 \\
210\end{array}$ & $\begin{array}{l}1 / \sqrt{2} \\
1\end{array}$ & $\begin{array}{l}-\pi / 4 \\
\pi\end{array}$ & $P 4_{3} 32$ & $\begin{array}{l}+[\cos X \cdot \cos Y \cdot \cos Z+\sin X \cdot \sin Y \cdot \sin Z]+[\sin 2 X \cdot \sin Y+\sin 2 Y \cdot \sin Z \\
+\sin X \cdot \sin 2 Z+\cos X \cdot \sin 2 Y+\cos Y \cdot \sin 2 Z+\sin 2 X \cdot \cos Z]=0\end{array}$ & $I 4_{1} 32$ & $P 4_{3} 32<(111)_{-\pi / 4}^{1 / \sqrt{2}}(210)_{\pi}^{1}>I 4_{1} 32$ \\
\hline$\left(Y Y_{x x x}\right)^{*}$ & $C(Y)$ & $\begin{array}{l}111 \\
210\end{array}$ & $\begin{array}{l}1 / \sqrt{2} \\
1\end{array}$ & $\begin{array}{l}3 \pi / 4 \\
\pi\end{array}$ & $P 4_{3} 32$ & $\begin{array}{l}-[\cos X \cdot \cos Y \cdot \cos Z+\sin X \cdot \sin Y \cdot \sin Z]+[\sin 2 X \cdot \sin Y+\sin 2 Y \cdot \sin Z \\
+\sin X \cdot \sin 2 Z+\cos X \cdot \sin 2 Y+\cos Y \cdot \sin 2 Z+\sin 2 X \cdot \cos Z]=0\end{array}$ & $I 4_{1} 32$ & $P 4_{3} 32<(111)_{3 \pi / 4}^{1 / \sqrt{2}}(210)_{\pi}^{1}>I 4_{1} 32$ \\
\hline$F_{x x x}{ }^{*}$ & ${ }^{ \pm} Y$ & $\begin{array}{l}111 \\
210\end{array}$ & $\begin{array}{l}1 \\
1\end{array}$ & $\begin{array}{l}0 \\
\pi\end{array}$ & $\operatorname{Pa} \overline{3}$ & $+2 \cdot \cos X \cdot \cos Y \cdot \cos Z+[\sin 2 X \cdot \sin Y+\sin X \cdot \sin 2 Z+\sin 2 Y \cdot \sin Z]=0$ & $I a \overline{3}$ & $\operatorname{Pa} \overline{3}<(111)_{0}^{1}(210)_{\pi}^{1}>\operatorname{Pa} \overline{3}$ \\
\hline$\left(F F_{x x x}\right)^{*}$ & $C\left({ }^{ \pm} Y\right)$ & $\begin{array}{l}111 \\
210\end{array}$ & $\begin{array}{l}1 \\
1\end{array}$ & $\begin{array}{l}\pi \\
\pi\end{array}$ & $P a \overline{3}$ & $-2 \cdot \cos X \cdot \cos Y \cdot \cos Z+[\sin 2 X \cdot \sin Y+\sin X \cdot \sin 2 Z+\sin 2 Y \cdot \sin Z]=0$ & $I a \overline{3}$ & $P a \overline{3}<(111)_{\pi}^{1}(210)_{\pi}^{1}>P a \overline{3}$ \\
\hline$F_{x x}-P_{2} F_{x}$ & $F-R D$ & $\begin{array}{l}111 \\
220\end{array}$ & $\begin{array}{l}2 \\
1\end{array}$ & $\begin{array}{l}0 \\
\pi\end{array}$ & $F m \overline{3} m$ & $4 \cdot \cos X \cdot \cos Y \cdot \cos Z-[\cos 2 X \cdot \cos 2 Y+\cos 2 X \cdot \cos 2 Z+\cos 2 Y \cdot \cos 2 Z]=0$ & $F m \overline{3} m$ & $F m \overline{3} m<(111)_{0}^{2}(220)_{\pi}^{1}>F m \overline{3} m$ \\
\hline$Q^{*}$ & $S T 1$ & 01.1 & 1 & 0 & $P 6_{2} 22$ & $(\cos X-2 \cos Y) \cdot \cos Z-\sqrt{3} \cdot \sin Z \cdot(\cos (X-Y)-\cos X)+\cos (X-Y) \cdot \cos Z=0$ & $P 6_{4} 22$ & $P 6_{2} 22<(011)_{0}^{1}>P 6_{4} 22$ \\
\hline
\end{tabular}



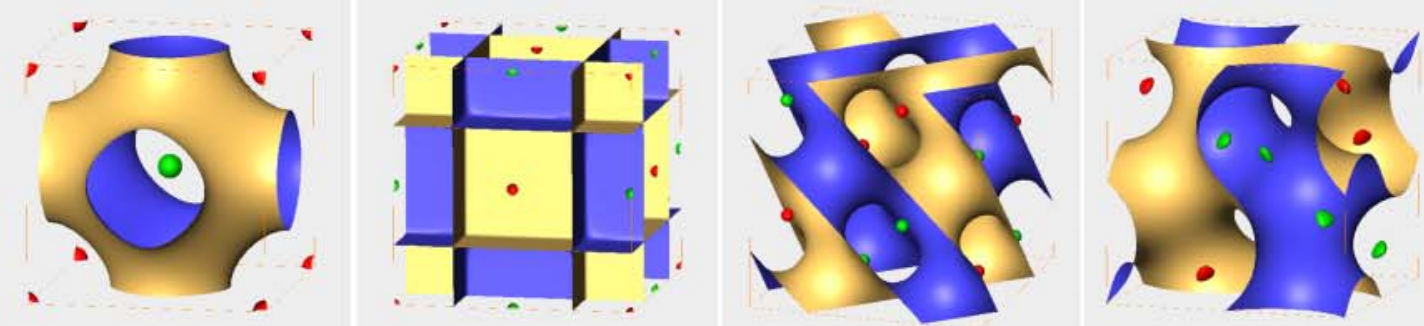

$P m \overline{3} m<(100)^{1}>I m \overline{3} m$

$F m \overline{3} m<(111)_{0}^{1}>P m \overline{3} m$

$F d \overline{3} m<(111)_{0}^{1}>P n \overline{3} m$

$I 4_{1} 32<(110)^{1}{ }_{\pi / 2}>I a \overline{3} d$
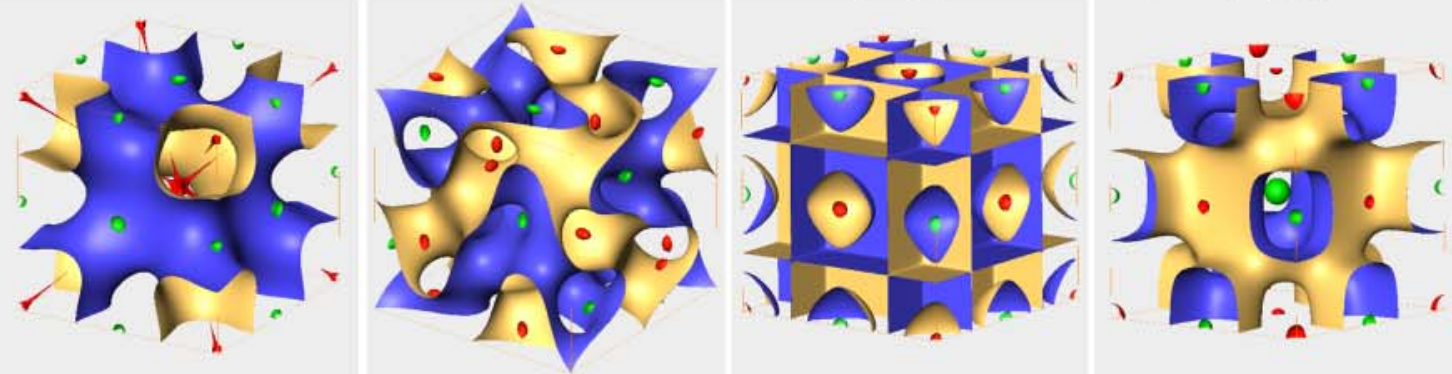

$\operatorname{Im} \overline{3} m<(110)_{0}^{1}$

$(200)^{1}{ }_{\pi}>\operatorname{Im} \overline{3} m$

$\overline{I 4} 3 d<(211)^{1}{ }_{\pi / 2}>I \bar{a} \overline{3} d$

$F d \overline{3} m<(311)_{0}^{1}>P n \overline{3} m$

$P m \overline{3} m<(100)^{1}{ }_{0}$
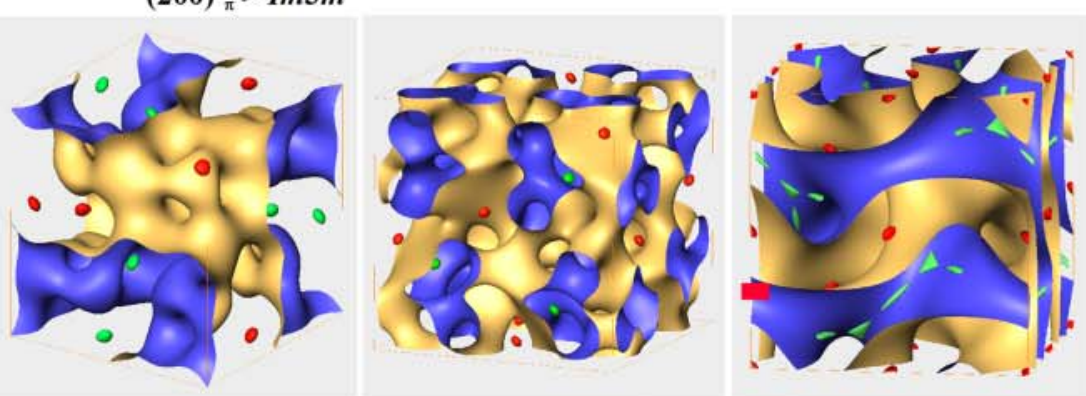

$(111)^{1}{ }_{0}>\operatorname{Im} \overline{3} m$

$I 4,32<(110)^{3}{ }_{\pi / 2}$

$(310)^{2}{ }_{\pi / 2}>I a \overline{3} d$

$I a \overline{3}<(200)^{2}{ }_{0}(321)^{1}{ }_{\pi}$

$$
I a \overline{3} d<(220)^{1}{ }_{0}
$$

$(211)^{1}{ }_{0}>I a \overline{3} d$

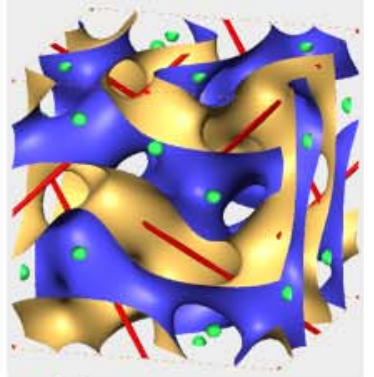

$I a \overline{3} d<(220)^{1}{ }_{0}$

$(231)^{1}{ }_{\pi}>I a \overline{3} d$

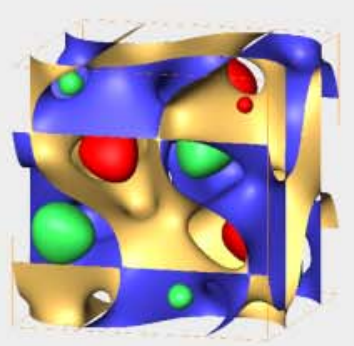

$(211)^{1}>I a \overline{3} d$

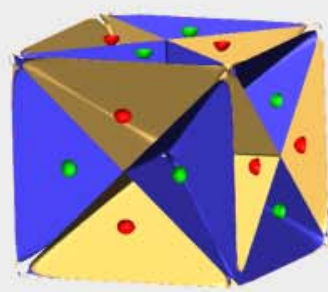

$$
\begin{aligned}
P 4_{3} 32< & (111)^{0.7}{ }_{-\pi / 4} \\
& (210)^{1}{ }_{\pi}>I 4_{1} 32
\end{aligned}
$$

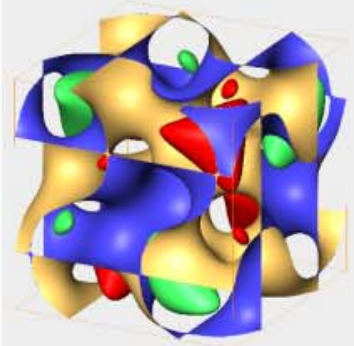

$\mathrm{P4}_{3} 32<(111)^{0.7}{ }_{3 \pi / 4}$

$(210)^{1}{ }_{\pi}>I 4_{1} 32$

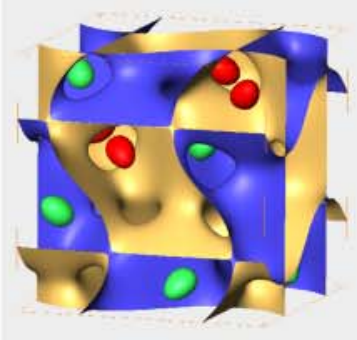

$P a \overline{3}<(111)^{1}{ }_{0}$

$(210)^{1}{ }_{\pi}>I a \overline{3}$

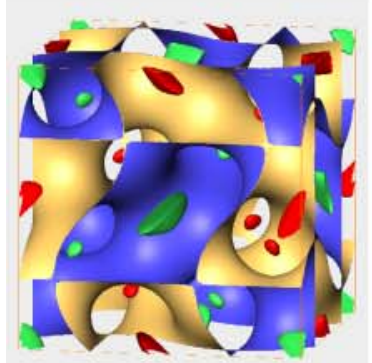

$$
P a \overline{3}<(111)^{1}{ }_{\pi}
$$

$(210)^{1}{ }_{\pi}>I \bar{a} \overline{3}$

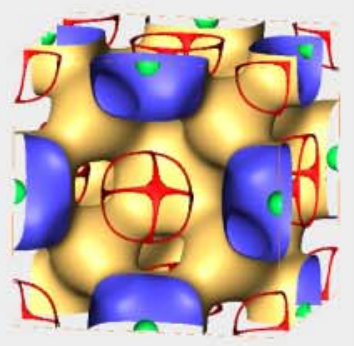

$F m \overline{3} \boldsymbol{m}<(111)^{2}{ }_{0}$

$(220)^{1}{ }_{\pi}>F m \overline{3} m$

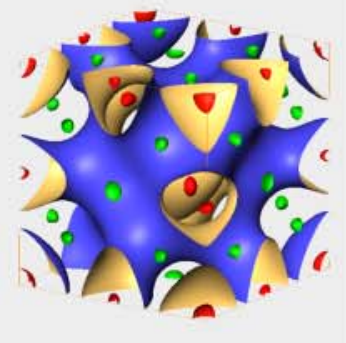

$P m \overline{3} m<(210)_{0}^{1}>\operatorname{Im} \overline{3} m$

Fig. 5. Shape of selected Periodic Nodal Surfaces and positions of the extreme points of the function $R(\mathbf{r})$ : yellow side of each points out to the positive values of $R(\mathbf{r})$, the blue side points out to the negative values; red isosurface visualizes the positions of the maximal, green isosurface shows the position of the minimal values of $R(\mathbf{r})$. 
short form $\mathbf{h}=h \mathbf{a}^{*}$ ) and $\vec{r}=x \mathbf{a}+y \mathbf{b}+z \mathbf{c}$ (in short form $\mathbf{r}=x \mathbf{a}$, respectively; $\alpha\left(\mathbf{h}_{n}\right)$ is the symmetry related phase shift of $S\left(\mathbf{h}_{n}\right)$.

In accordance with the definition above, the complete symbol of a PNS, for example

$$
I 4 / m c m<(110)_{0}^{1} ;(121)_{\pi}^{0.2}>I 4 / m c m,
$$

starts with the generating space group symmetry, followed by brackets \langle\rangle and ends with the resulting PNS space group symmetry [36, 37]. The brackets \langle\rangle contain the information about the representative vectors $(h k l)_{\alpha}^{|S|}$ with their phases and amplitudes that have to be used in the Fourier transform in order to develop the desired PNS. This set is derived by permutation of $h k l$ according tospace group symmetry.

The ease of deriving PNS by the help of selected sets of structure factors $S(\mathbf{h})$ raised the question of how to select those $S$ which are characteristic for a given space group. This was insofar important as the extinction conditions alone are not sufficient to decide on this problem because a low-indexed $S(h k l, \alpha)$ set may generate a higher symmetry than the space group in question. So for all cubic body centered groups the sets $S(110, \alpha)$ are only characteristic for $\operatorname{Im} \overline{3} m$ and for $I 4_{1} 32$, respectively, discriminated by the value of $\alpha$. The first characteristic set for $I 432$ as well as for $I 23$ is $S(321, \alpha)$ only. It is even more surprising that for the cubic primitive groups $P n \overline{3} n, P n \overline{3}$ and $P 23$ no single characteristic structure factor is found with shorter $/ \mathbf{h} /$ than $S(421, \alpha)$ ! A complete survey of characteristic sets was worked out by von Schnering however not yet published completely [38]. The shape of the selected PNS and the positions of the extreme points of the according sum functions are presented in Fig. 5.

\section{PNS, exponential scale and other approaches}

S. Anderson formulated the concept of shape description using exponential scale equations and applied it on crystal shapes [39], cubosomes [40], basic sphere packings and fundamental crystal structures [40-43]. According to his approach, objects with translational symmetry can be described in exponential form (ES) with the following equations or their combinations:

$$
\begin{aligned}
& \operatorname{ES} 1(\mathbf{r})=\sum_{n=1}^{N} \exp \left(2 \pi \mathbf{h}_{n} \mathbf{r}\right) \\
& \operatorname{ES} 2(\mathbf{r})=\exp \left(\sum_{n=1}^{N} \exp \left(2 \pi \mathbf{h}_{n} \mathbf{r}\right) .\right.
\end{aligned}
$$

Here $\mathbf{h}_{n}$ and $\mathbf{r}$ are vectors in reciprocal and direct space, and $N$ is the number of reciprocal vectors used for summation. For the description of given objects, the isosurfaces of ES1 or/and ES2 with appropriate values were utilized.

The zero-electrostatic-potential areas separate the positively and negatively charged domains within an array of electric charges, e.g. a crystal structure. These PSPs are called Periodic Zero Potential Surfaces (P0PS) or Zero Equipotential Surfaces (ZEPS). For the last definition a generation method was developed by means of Jacobi the- ta functions $[44,45]$. Mathematically, this representation is close to the exponential surfaces introduced by Sten Andersson.

More for practical reasons A. L. Mackay developed a method for numerical binding of the minimal surface [46]. The calculation procedure starts with a table of the $z$ coordinates (as a function of $x$ any $y$ ) of an approximate surface, e.g. a PNS. Then in each point $(x, y)$ the first and second derivatives of $z$ with respect of $x$ and $y$ are calculated. They are further implemented in the Laplace-Young equation for the calculation of a new $z$ value which gives a new surface with the divergence value closer to zero. These operations are repeated until convergence criteria with respect of the area and integrated Gaussian curvatures are achieved. The integrated Gaussian curvature obtained from the boundary conditions serves as a check on the whole calculation. On this way a final table of $x, y, z$ coordinates of the points of a minimal surface is produced.

In some cases, the isovalues of exponential scale (ES) equations reveal surfaces, which topologically are very similar to the PNS. So, implementing the vector (100) with its equivalents for the cubic symmetry into the equation ES1 and using 7.56 as the function value gives a isosurface ([40], p. $85 \mathrm{ff}$ ) which has similar topology to the PNS $\operatorname{Pm} \overline{3} m<(100)>\operatorname{Im} \overline{3} m$, which is, in turn, very similar to the minimal surface $P$ [47]. A detailed analysis of both systems of surface generation (PNS and exponential scale) reveals their immanent relation. The ES equations in general can be represented as weighted sums of the PNS equations either with the multiplied reciprocal vectors $m \mathbf{h}_{n}$ or as weighted sum of the PNS equations raised to the power of $m$, where $m$ is an integer value equal to the number of the summands reduced by 1 . So, the shape of the isosurface with $\mathrm{ES} 1=81 N / 64$ is identical to that of the weighted sum of PNS's with the multiplied reciprocal vectors, $m \boldsymbol{h}_{n}$. An interesting by-product of the analysis above was the conclusion that the extreme values for both equations types can not be found analytically but only by the numerical way [48].

\section{Minimal structure factor sets defining structure types}

Taking the list of known structure types it is quite interesting to investigate what would be the smallest number of fundamental sets $S\left(\mathbf{h}_{n}\right)$ to discriminate all structures of a specific symmetry group. Let us choose cubic face centered structure types belonging to the diamond group $F d \overline{3} m$ [49] as listed in Pearson's Handbook [50]. Of course, the basic PNS which can be generated in the space group of diamond is of general validity for all the structure types that crystallize with that symmetry. In that sense, PNS may serve as an important tool in understanding the relations of those structures in a hierarchical sense, that is with increasing complexity. However, the fact that the level of the description has not taken into account the atomic sites imposes some extensions of the concept and some other point of view, if we want to understand which variations are permitted around a basic organizer such as the $D^{*}$ surface. If the zinc-blende structure is the "natural" realiza- 
Table 2. Structure types with diamond group symmetry and their characteristic sets of structure factors $S\left(\mathbf{h}_{n}\right)$. In lines: amplitudes $|\mathbf{S}|$ and signs/phase angles, $+\rightarrow \alpha=0^{\circ},-\rightarrow \alpha$ $=180^{\circ}$.

\begin{tabular}{|c|c|c|c|c|c|c|c|c|}
\hline $\begin{array}{l}\text { Structure } \\
\text { type }\end{array}$ & 111 & 220 & 222 & 311 & 331 & 333 & 400 & 422 \\
\hline $\begin{array}{l}c F 16 \\
\mathrm{NaTl}\end{array}$ & 0 & $\begin{array}{l}1 \\
-\end{array}$ & 0 & 0 & 0 & 0 & $\begin{array}{l}1 \\
-\end{array}$ & $\begin{array}{l}1 \\
+\end{array}$ \\
\hline $\begin{array}{l}c F 24 \\
\mathrm{MgCu}_{2}\end{array}$ & $\begin{array}{c}0.10 \\
-\end{array}$ & $0.33-$ & $\begin{array}{c}0.67 \\
+\end{array}$ & $\begin{array}{c}0.57 \\
+\end{array}$ & $\begin{array}{c}0.10 \\
+\end{array}$ & $\begin{array}{c}0.57 \\
-\end{array}$ & $\begin{array}{c}0.33 \\
+\end{array}$ & $\begin{array}{c}0.33 \\
+\end{array}$ \\
\hline $\begin{array}{l}c F 32 \\
\mathrm{CsNbN}_{2}\end{array}$ & $\begin{array}{c}0.25 \\
-\end{array}$ & $\begin{array}{c}0.50 \\
-\end{array}$ & $\begin{array}{c}0.50 \\
+\end{array}$ & $\begin{array}{c}0.25 \\
+\end{array}$ & $\begin{array}{l}0.25 \\
+\end{array}$ & $\begin{array}{c}0.25 \\
-\end{array}$ & 0 & $\begin{array}{c}0.50 \\
+\end{array}$ \\
\hline $\begin{array}{l}c F 48 \\
\mathrm{TiS}_{2}\end{array}$ & $\begin{array}{c}0.20 \\
-\end{array}$ & $\begin{array}{l}0 \\
-\end{array}$ & $\begin{array}{c}0.33 \\
-\end{array}$ & $\begin{array}{c}0.16 \\
+\end{array}$ & $\begin{array}{c}0.12 \\
+\end{array}$ & $\begin{array}{c}0.07 \\
-\end{array}$ & $\begin{array}{l}1 \\
+\end{array}$ & $\begin{array}{l}0 \\
-\end{array}$ \\
\hline $\begin{array}{l}c F 56 \\
\text { spinel }\end{array}$ & $\begin{array}{c}0.16 \\
-\end{array}$ & $\begin{array}{c}0.19 \\
-\end{array}$ & $\begin{array}{c}0.22 \\
-\end{array}$ & $\begin{array}{c}0.20 \\
+\end{array}$ & $\begin{array}{c}0.13 \\
-\end{array}$ & $\begin{array}{c}0.07 \\
+\end{array}$ & $\begin{array}{c}0.63 \\
+\end{array}$ & $\begin{array}{c}0.11 \\
+\end{array}$ \\
\hline $\begin{array}{l}c F 64 \\
\mathrm{Li}_{2} \mathrm{TiS}_{4}\end{array}$ & $\begin{array}{c}0.02 \\
+\end{array}$ & 0 & 0 & $\begin{array}{c}0.10 \\
+\end{array}$ & 0 & $\begin{array}{c}0.10 \\
-\end{array}$ & $\begin{array}{l}1 \\
+\end{array}$ & 0 \\
\hline $\begin{array}{l}c F 80 \\
\mathrm{As}_{2} \mathrm{O}_{3}\end{array}$ & $\begin{array}{c}0.28 \\
-\end{array}$ & $\begin{array}{c}0.10 \\
-\end{array}$ & $\begin{array}{c}0.36 \\
-\end{array}$ & $\begin{array}{c}0.02 \\
-\end{array}$ & $\begin{array}{c}0.33 \\
-\end{array}$ & $\begin{array}{c}0.21 \\
-\end{array}$ & $\begin{array}{c}0.01 \\
+\end{array}$ & $\begin{array}{c}0.32 \\
-\end{array}$ \\
\hline $\begin{array}{l}c F 80 \\
\mathrm{ThZr}_{2} \mathrm{H}_{7}\end{array}$ & $\begin{array}{c}0.10 \\
-\end{array}$ & 0 & $\begin{array}{c}0.20 \\
+\end{array}$ & $\begin{array}{c}0.10 \\
+\end{array}$ & $\begin{array}{c}0.10 \\
+\end{array}$ & $\begin{array}{c}0.10 \\
-\end{array}$ & $\begin{array}{c}0.60 \\
-\end{array}$ & 0 \\
\hline $\begin{array}{l}c F 96 \\
\mathrm{Ti}_{2} \mathrm{Ni}\end{array}$ & $\begin{array}{c}0.06 \\
-\end{array}$ & $\begin{array}{c}0.006 \\
+\end{array}$ & $\begin{array}{c}0.071 \\
-\end{array}$ & $\begin{array}{l}0.014 \\
+\end{array}$ & $\begin{array}{c}0.133 \\
+\end{array}$ & $\begin{array}{c}0.472 \\
+\end{array}$ & $\begin{array}{c}0.026 \\
+\end{array}$ & $\begin{array}{c}0.274 \\
-\end{array}$ \\
\hline $\begin{array}{l}c F 120 \\
\mathrm{Mn}_{4} \mathrm{Co}_{7} \mathrm{Sb}_{4}\end{array}$ & $\begin{array}{c}0.056 \\
-\end{array}$ & $\begin{array}{c}0.061 \\
+\end{array}$ & $\begin{array}{c}0.0093 \\
+\end{array}$ & $\begin{array}{c}0.034 \\
-\end{array}$ & $\begin{array}{l}0.033 \\
+\end{array}$ & $\begin{array}{c}0.072 \\
-\end{array}$ & $\begin{array}{c}0.072 \\
+\end{array}$ & $\begin{array}{c}0.072 \\
-\end{array}$ \\
\hline $\begin{array}{l}c F 128 \\
\mathrm{Li}_{13} \mathrm{In}_{3}\end{array}$ & $\begin{array}{c}0.024 \\
-\end{array}$ & $\begin{array}{c}0.004 \\
-\end{array}$ & $\begin{array}{c}0.006 \\
+\end{array}$ & $\begin{array}{c}0.008 \\
-\end{array}$ & $\begin{array}{c}0.04 \\
-\end{array}$ & $\begin{array}{c}0.07 \\
+\end{array}$ & $\begin{array}{c}0.008 \\
-\end{array}$ & $\begin{array}{c}0.004 \\
-\end{array}$ \\
\hline $\begin{array}{l}c F 128 \\
\mathrm{BaGe}_{2} \mathrm{~S}_{5}\end{array}$ & $\begin{array}{c}0.06 \\
+\end{array}$ & $\begin{array}{c}0.112 \\
-\end{array}$ & $\begin{array}{c}0.194 \\
-\end{array}$ & $\begin{array}{l}0.055 \\
+\end{array}$ & $\begin{array}{l}0.198 \\
+\end{array}$ & $\begin{array}{l}0.200 \\
+\end{array}$ & $\begin{array}{c}0.160 \\
+\end{array}$ & $\begin{array}{c}0.248 \\
-\end{array}$ \\
\hline $\begin{array}{l}c F 128 \\
\mathrm{Ti}_{2} \mathrm{NiH} \\
\text { as } \mathrm{Ti}_{2} \mathrm{Ni}\end{array}$ & $\begin{array}{l}0.015 \\
+ \\
-\end{array}$ & $\begin{array}{c}0.120 \\
- \\
+\end{array}$ & $\begin{array}{c}0.071 \\
+ \\
-\end{array}$ & $\begin{array}{c}0.052 \\
- \\
+\end{array}$ & $\begin{array}{c}0.037 \\
+ \\
+\end{array}$ & $\begin{array}{l}0.416 \\
+ \\
+\end{array}$ & $\begin{array}{l}0.020 \\
+ \\
+\end{array}$ & $\begin{array}{l}0.080 \\
- \\
-\end{array}$ \\
\hline $\begin{array}{l}c F 144 \\
\mathrm{Dy}_{5} \mathrm{Pd}_{2}\end{array}$ & $\begin{array}{c}0.21 \\
-\end{array}$ & $\begin{array}{c}0.158 \\
-\end{array}$ & $\begin{array}{c}0.031 \\
-\end{array}$ & $\begin{array}{c}0.053 \\
-\end{array}$ & $\begin{array}{c}0.199 \\
+\end{array}$ & $\begin{array}{c}0.315 \\
+\end{array}$ & $\begin{array}{c}0.046 \\
+\end{array}$ & $\begin{array}{c}0.108 \\
-\end{array}$ \\
\hline $\begin{array}{l}c F 160 \\
\mathrm{Na}_{24} \mathrm{Si}_{136}\end{array}$ & $\begin{array}{c}0.062 \\
-\end{array}$ & $\begin{array}{c}0.058 \\
+\end{array}$ & $\begin{array}{c}0.072 \\
-\end{array}$ & $\begin{array}{c}0.039 \\
-\end{array}$ & $\begin{array}{c}0.052 \\
-\end{array}$ & $\begin{array}{c}0.258 \\
-\end{array}$ & $\begin{array}{c}0.101 \\
-\end{array}$ & $\begin{array}{l}0.157 \\
+\end{array}$ \\
\hline $\begin{array}{l}c F 184 \\
\mathrm{~V}_{2} \mathrm{Al}_{20}\end{array}$ & $\begin{array}{l}0.044 \\
+\end{array}$ & $\begin{array}{c}0.013 \\
+\end{array}$ & $\begin{array}{c}0.007 \\
+\end{array}$ & $\begin{array}{c}0.023 \\
-\end{array}$ & $\begin{array}{c}0.007 \\
-\end{array}$ & $\begin{array}{c}0.028 \\
-\end{array}$ & $\begin{array}{c}0.012 \\
+\end{array}$ & $\begin{array}{c}0.034 \\
-\end{array}$ \\
\hline $\begin{array}{l}c F 216 \\
\mathrm{Li}_{2} \mathrm{P}_{2} \mathrm{~N}_{5}\end{array}$ & $\begin{array}{c}0.22 \\
-\end{array}$ & $\begin{array}{c}0.115 \\
-\end{array}$ & $\begin{array}{c}0.031 \\
-\end{array}$ & 0 & $\begin{array}{c}0.04 \\
+\end{array}$ & $\begin{array}{c}0.04 \\
-\end{array}$ & $\begin{array}{c}0.53 \\
+\end{array}$ & $\begin{array}{c}0.23 \\
-\end{array}$ \\
\hline $\begin{array}{l}c F 464 \\
\mathrm{Na}_{35} \mathrm{Cd}_{24} \mathrm{Ga}_{56}\end{array}$ & $\begin{array}{c}0.031 \\
+\end{array}$ & $\begin{array}{c}0.011 \\
-\end{array}$ & $\begin{array}{c}0.009 \\
+\end{array}$ & $\begin{array}{c}0.017 \\
-\end{array}$ & $\begin{array}{c}0.012 \\
-\end{array}$ & $\begin{array}{c}0025 \\
-\end{array}$ & $\begin{array}{c}0.009 \\
+\end{array}$ & $\begin{array}{c}0.005 \\
-\end{array}$ \\
\hline $\begin{array}{l}c F 464 \\
\mathrm{Na}_{17} \mathrm{Ga}_{29} \mathrm{In}_{12}\end{array}$ & $\begin{array}{l}0.051 \\
+\end{array}$ & $\begin{array}{c}0.01 \\
-\end{array}$ & $\begin{array}{c}0.009 \\
+\end{array}$ & $\begin{array}{c}0.023 \\
-\end{array}$ & $\begin{array}{c}0.011 \\
-\end{array}$ & $\begin{array}{c}0.027 \\
-\end{array}$ & $\begin{array}{c}0.005 \\
+\end{array}$ & $\begin{array}{c}0.008 \\
-\end{array}$ \\
\hline $\begin{array}{l}c F 1192 \\
\mathrm{NaCd}_{2}\end{array}$ & $\begin{array}{l}0.037 \\
+\end{array}$ & $\begin{array}{c}0.003 \\
+\end{array}$ & $\begin{array}{c}0.012 \\
+\end{array}$ & $\begin{array}{c}0.006 \\
-\end{array}$ & $\begin{array}{c}0.095 \\
-\end{array}$ & $\begin{array}{c}0.001 \\
-\end{array}$ & $\begin{array}{c}0.004 \\
+\end{array}$ & $\begin{array}{l}0.016 \\
+\end{array}$ \\
\hline $\begin{array}{l}c F 1832 \\
\mathrm{Mg}_{2} \mathrm{Al}_{3}\end{array}$ & $\begin{array}{c}0.172 \\
-\end{array}$ & $\begin{array}{c}0.112 \\
-\end{array}$ & $\begin{array}{c}0.011 \\
+\end{array}$ & $\begin{array}{c}0.04 \\
-\end{array}$ & $\begin{array}{l}0.009 \\
+\end{array}$ & $\begin{array}{l}0.052 \\
+\end{array}$ & 0 & $\begin{array}{c}0.004 \\
+\end{array}$ \\
\hline
\end{tabular}

tion of the symmetry recipe of the $D^{*}$ surface, the so-called aristotype, what about other representatives of this symmetry like the spinel type or some extremely complex one like that of $\mathrm{Mg}_{2} \mathrm{Al}_{3}$ ?
To be more precise, out of the large number of structures crystallize with space group $F d \overline{3} m$, only the representative variants of the structure types were chosen for comparison. The structure types were then compared on 
the basis of the occupied positions which were all represented by one atom type (carbon). The development of complexity was worked out by using the PNS approach under preservation of the underlying basic topological essence of the group. The calculation of the structure factors was then performed according to the following sequence:

1. Standardize all structure types to one setting [19].

2. Generate all the positions and assign a uniform scattering factor (carbon).

3. Calculate a scale factor according to $S(000)$, which corresponds to the total number of positions.

4. Generate a sufficiently large number of $S\left(\mathbf{h}_{n}\right)$ of shortest $\mathbf{h}_{n}$ length, i.e. increasing Miller indices $h k l$, which are close to the centre of reciprocal space and carry general topological information.

5. Only consider $S\left(\mathbf{h}_{n}\right)$ with large amplitudes.

6. Finally, the size of the complete set of $S\left(\mathbf{h}_{n}, \alpha\right)$ was reduced with respect to the length of $\mathbf{h}_{n}$ in order to find the smallest set which allows us to discriminate all investigated structure types by their phase sets alone.

The result is that a series of eight sets $S\left(\mathbf{h}_{n}, \alpha\right)$ with smallest $\mathbf{h}_{n}$ vectors is sufficiently large to discriminate structure types from $c F 16$ and $c F 1832$, from 16 to 1832 atoms per unit cell! There is only one exception which is the two $c F 464$ types which are differently classified in data bases and look quite different concerning their crystal structure plots (Table 2). However, comparison of the Wyckoff sites reveals that they are practically isostructural.

This means that very few basic $S\left(\mathbf{h}_{n}, \alpha\right)$ sets allow to discriminate topologically different frameworks of the same symmetry. Furthermore, the 8 structure factor sets of Table 2 generate for each of the 20 structure types a different separable PNS.

\section{Use for structure determination}

Applying a larger number of reflections as it usually necessary for a PNS, an envelope surface may be generated which roughly describes the shape of the protein molecule and defines the boundary between the molecule and the solvent [50]. Such surfaces and a PNSs are mathematically easily related, despite a PNS usually does not have a closed form. This similarity was reason enough to adopt the term 'structure envelope' also for PNS [51].

Applying a special algorithm for the sensible phase determination for the generating reflections, the resulting PNS was hoped to describe in zero approximation the electron density in the crystal structure determined. Combining this result with any of the direct-space methods of crystal structure determination, the variety of the possible structure-fragment positions, orientations and conformations may be strongly reduced. This technique was successfully applied to the determination of the crystal structures of zeolites and molecular compounds [52].

The maxima and the minima of the PNS equation reflect the positions suitable for the structural units with opposite charges. This feature may be used for generating of a starting model for crystal structure determination routines, which are not based on traditional direct methods [53].

\section{Geometry, topology, and bonding}

Periodic Nodal Surfaces generated from the shortest Bragg vectors $\boldsymbol{h}$ in reciprocal space reveal different features of the structural relationships or atomic interactions. As for atomic interactions, a PNS either emphasizes that part of the space where a distinct kind of chemical bonding takes place or separates of the space parts with different kinds of predominant chemical bonding. In the simplest case a PNS separates the cations and the anions in a crystal structure: the often referenced so-called $P$ surface $\operatorname{Pm} \overline{3} m<(100)_{0}^{1}>\operatorname{Im} \overline{3} m$ separates ions of different charge in the CsCl-type crystal structures (Fig. 6a). In the more complex crystal structures of intermetallic clathrates $\alpha-\mathrm{Eu}_{8} \mathrm{Ga}_{16} \mathrm{Ge}_{30}, \beta-\mathrm{Eu}_{8} \mathrm{Ga}_{16} \mathrm{Ge}_{30}$ [54], and $\mathrm{Ba}_{6} \mathrm{Ge}_{25}$ [55], an appropriate PNS separates the cations located in the cavities of the framework anions, revealing the interaction between the both structure components (Fig. 6b-d). This general feature is further recognizable in the case of both modifications of $\mathrm{BaAl}_{2} \mathrm{Ge}_{2}$. In (a)

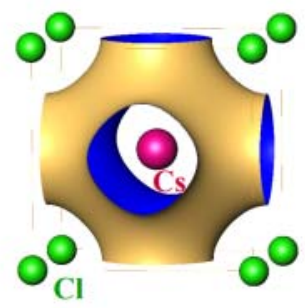

CsCl

$P m \overline{3} m<(100)_{0}^{1}>P m \overline{3} m$

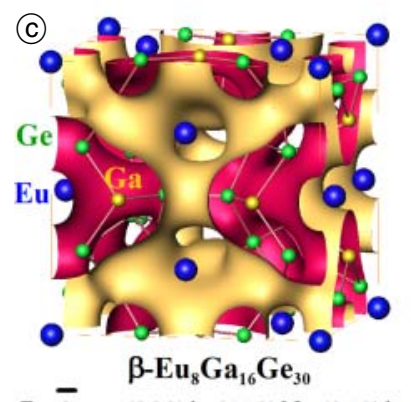

$P m \overline{3} n<(200)_{0}^{1}(110)^{0.8}{ }_{0}(210)^{1}$

(e)

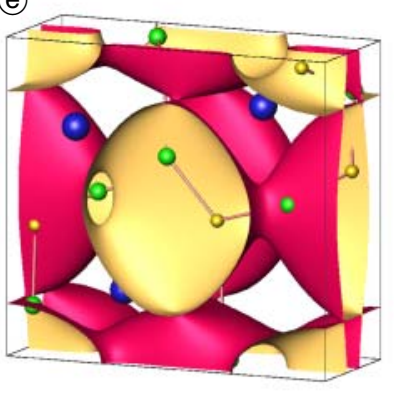

$\alpha-\mathrm{BaAl}_{2} \mathrm{Ge}_{2}$

Pnma $<(002)^{1}{ }_{0}(200)^{2}{ }_{0}$

$(011)_{\pi}^{2}(111)^{2}>$ Pnma
$(211)^{0.5}{ }_{0}(222)_{0}^{1}>P m 3 n$

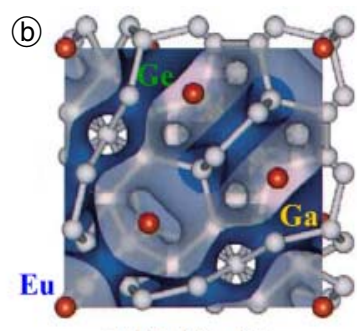

$\alpha-\mathbf{E u}_{8} \mathrm{Ga}_{16} \mathrm{Ge}_{30}$

$I \overline{4} m<(200)^{2}{ }_{\pi}(110)^{0.5}{ }_{0}$

(d)

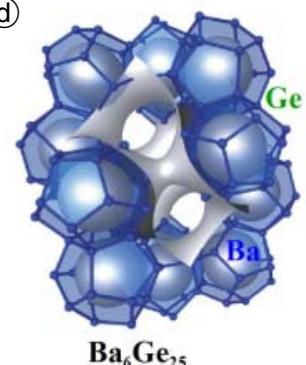

$\mathrm{Ba}_{6} \mathrm{Ge}_{25}$ $(220)^{1}{ }_{\pi}(211)_{\pi / 2}^{1}>I \overline{43} m$

$P 4_{1} 31<(110)^{0.578}{ }_{\pi / 2}(111)^{1}{ }_{\pi / 4}>P 4_{1} 32$

(f)

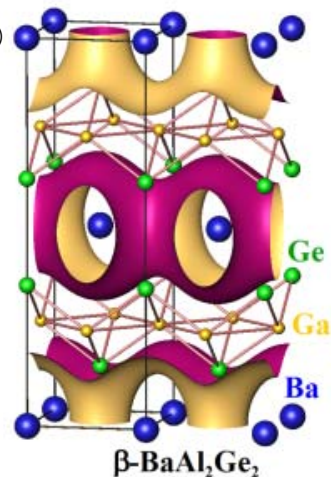

$I 4 / m m m<(101)_{0}^{1}(002)^{2}{ }_{0}>I 4 / m m m$

Fig. 6. Periodic Nodal Surfaces separating differently charged regions in the crystals structures of $\mathrm{CsCl}(\mathbf{a}), \alpha-\mathrm{Eu}_{8} \mathrm{Ga}_{16} \mathrm{Ge}_{30}$ [54] (b), $\beta$ $\mathrm{Eu}_{8} \mathrm{Ga}_{16} \mathrm{Ge}_{30}$ [54] (c), $\mathrm{Ba}_{6} \mathrm{Ge}_{25}$ [55] (d), $\alpha$ - and $\beta$ - $\mathrm{BaAl}_{2} \mathrm{Ge}_{2}$ [56] (e), (f). 
the $\beta$ polytype PNS separates the cations from the layered anion, in the $\alpha$ polytype a topologically similar PNS separates a three-dimensional anion from the embedded cations (Fig. 6e, f [56]).

A very good measure for atomic interactions which can be compared by PNS is given by the electron localizability approach [57-61, 108]. By topological analysis of the Electron Localizability Indicator (or Electron Localization Function) different atomic interactions can be indentified and ascribed to the distinct space parts within a crystal structure. An appropriate PNS separates the structure regions with different chemical interactions. A prominent example was found in $\mathrm{RhBi}_{4}[62,63]$. The regions with the covalent $\mathrm{Rh}-\mathrm{Bi}$ interactions forming hyperbolic layers are separated from the regions with the lone-pair interactions between these layers by the Giroid PNS (Fig. 7a). In the structurally related intermetallic compounds $\mathrm{CuAl}_{2}$ [64] and $\mathrm{PdGa}_{5}[28,66]$, the transition metals are coordinated by $\mathrm{Al}$ or $\mathrm{Ga}$ forming tetragonal antiprisms. Within these antiprisms, the multicentre bonding is prevalent. Between the antiprisms, the two-centre $\mathrm{Al}-\mathrm{Al}$ or $\mathrm{Ga}-\mathrm{Ga}$ bonds are formed. Both regions with different bonding kind are separated by an PNS (Fig. 7b, c). In the recently found binary compound $\mathrm{Mg}_{3} \mathrm{Ru}_{2}$, ruthenium atoms form a 3D polyanion, which is separated by a PNS from the magnesium substructure [67]. Besides the ionic interactions between both substructures, the PNS visualizes the geometric place for the multicentre interaction between magnesium and ruthenium (Fig. 7d). A special feature of the defect clathrate-I structure of $\mathrm{Ba}_{8} \mathrm{Ge}_{43} \square_{3}$ are the lone pairs of Ge around the defects in the ordered superstructure [68]. Also in such topologically extremely complex situation is it possible to visualize the arrangement of the lone pairs by help of a PNS (Fig. 7e).

Interestingly there is an approach in plane wave theory to explain structural stability which relates somehow to the concept of PNS. One uses the Fourier transform of the pair correlation function which is a function of distance but isotropic in 3D and thus structure independent, and reads it out at $\boldsymbol{h}$ vectors of the principle structure factors (closest to the origin of reciprocal space $\Gamma$ ). Only structures having structure factor sets which can read out considerable negative energy contributions from the continuous pair correlation function are stable. This was used long time ago by Heine et al. to explain the stability of the $\alpha$-gallium structure over an $f c c$ lattice (Fig. 8).

These days, it is quite obvious, that in a plane wave expansion of the wave function of a crystalline solid, the major energy contributions will be found by those plane waves which correspond to strong principle structure factors. ${ }^{2}$ However, little work has been dedicated to correlations of single plane waves or plane wave sets with actual structural features, up to now. In a recent paper, H. Aoki et al. derive a Schrödinger-like equation for PMS, calculate band structures and show principle plane wave states [70]. Though very interesting and in a way fundamental

\footnotetext{
2 In case of different atom types with large differences in electron number the argument better applies to the so-called normalized structure factors $\boldsymbol{E}$ which are more or less free of the influence of form factors or scattering curve differences of the individual atoms.
}

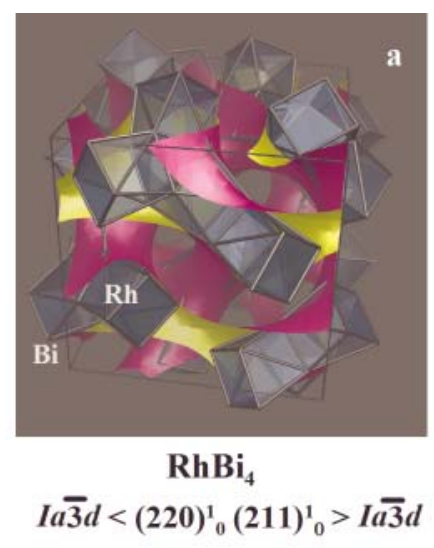

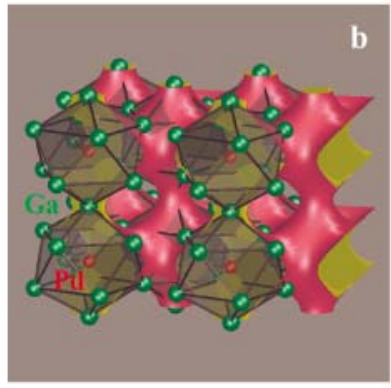

$\mathrm{PdGa}_{5}$

$I 4 / m c m<(002)^{1}$

$(110)^{0.83}>I 4 / m c m$

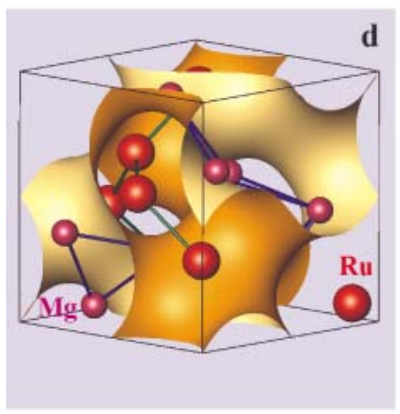

$$
\begin{gathered}
\mathrm{Mg}_{3} \mathrm{Ru}_{2} \\
P 4_{1} 31<(110)^{1}{ }_{\pi}>P 4_{1} 32
\end{gathered}
$$

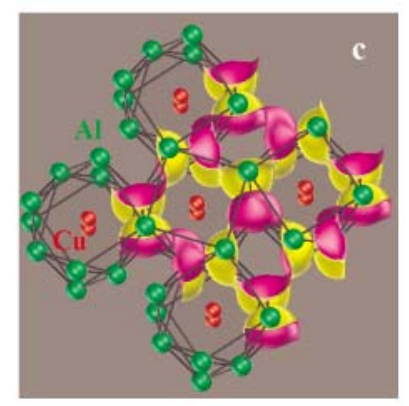

$\mathrm{CuAl}$

$I 4 / m c m<(110)^{1}$

$(121)^{0.2}>I 4 / \mathrm{mcm}$

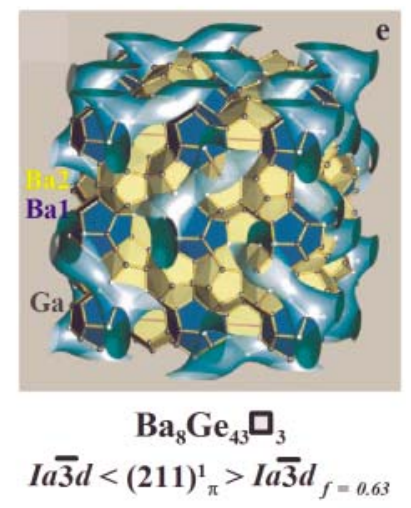

Fig. 7. Periodic Nodal Surfaces separating regions with different chemical interactions in the crystal structures: (a) covalent $\mathrm{Rh}-\mathrm{Bi}$ interactions within the tetragonal antiprisms $\left[\mathrm{RhBi}_{8}\right]$ and van-der-Waals interactions between the hyperbolic layers of antiprisms [62, 63]; (b), (c) multicentre $\mathrm{Pd}-\mathrm{Ga}$ and $\mathrm{Cu}-\mathrm{Al}$ interactions within the antiprisms $\left[\mathrm{PdGa}_{10}\right]$ and $\left[\mathrm{CuAl}_{8}\right]$ and two-centre bonds between the antiprisms in $\mathrm{PdGa}_{5}$ [28] (b) and $\mathrm{CuAl}_{2}$ [64] (c); (d) $\mathrm{Ru}-\mathrm{Ru}$ interaction in the polyanionic part and magnesium cations in $\mathrm{Mg}_{3} \mathrm{Ru}_{2}$ [66]; (e) lone pair arrangements around the defects in the Ge framework and the main part of the framework in $\mathrm{Ba}_{8} \mathrm{Ge}_{43} \square_{3}$ [67].

for PNS research, this contribution, is more a mathematical than a physical one because the states and bands being derived are basically representations of the PMS in the group theoretical sense. Unless concrete electron numbers and effective potentials are specified, direct physical meaning must be lacking. Analyzing their band structure for Schwarz's $P$ surface in Fig. 9 we find that the bands belong to only two sets through their degeneracy at certain $\mathbf{k}$ vectors. The lower energy bands crossing points $a-e$ constitute a set of six bands which are folded by the smaller 

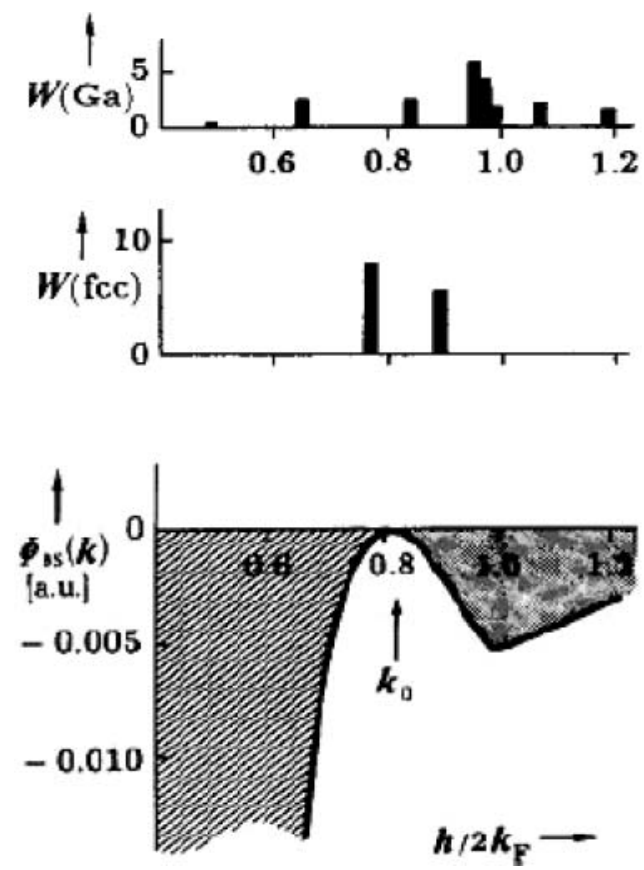

Fig. 8. (top) $\mathbf{k}$ dependence of structure factors for the $\alpha$-gallium and the alternative $f c c$ arrangement; (bottom) Fourier transform of pair correlation function for $\mathrm{Ga}-\mathrm{Ga}$ interactions. According Heine and Weaire, that structure which assembles most energy from the pair potential curve through its $\mathbf{k}$-vectors or structure factors $\mathbf{h}_{n}$ is the more stable one [68].
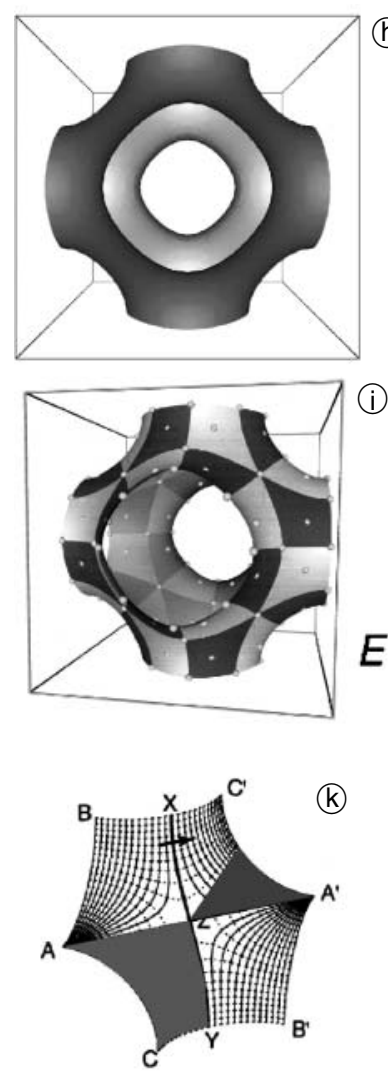

(1)

(i)

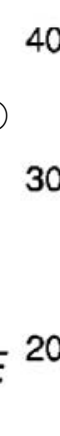

)

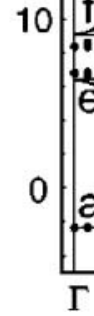

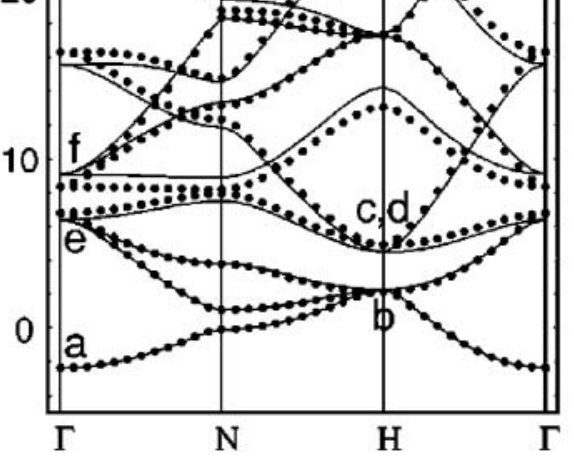
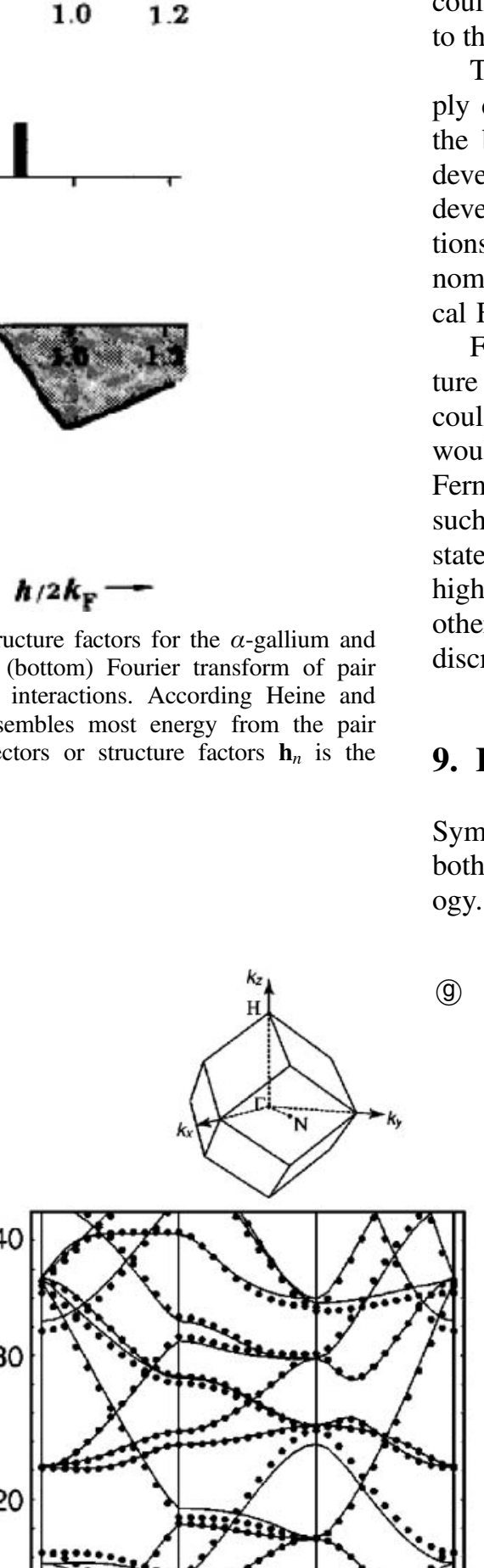

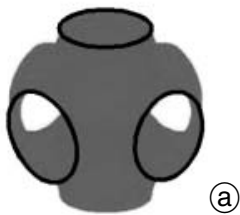

(a)
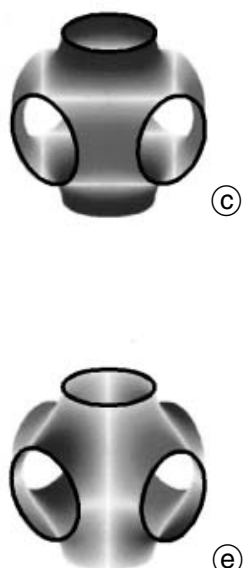

(e)
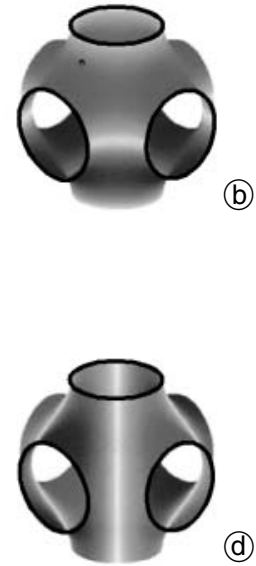

(b)

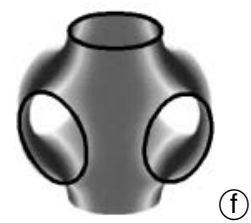

Fig. 9. Band structure of the $P$ surface after [70]: (a)-(f) wavefunction for $k$ points given in the band structure (g), (h) PNS of the $P$ surface; (i) the 48 fundamental tiles of the $P$ surface shown as a modulated PNS according to section 8; (k) unit cell of the band structure (g, in grey) and smaller cells unfolding the six-fold (blue) and the twelve-fold degenerate bands (red). 
of arrangements in space, like for example atoms in a structure. Every structural scientist knows that the notion of the atomic sites leaves a lot of freedom for interpretation of the way in which atoms are kept together to arrange as they do. The question of chemical bonding on local and on global scales is a wide field of interpretation and modeling at different levels of sophistication. Ab initio methods have the great appeal that they do not impose many constraints on the way atoms would rearrange if boundary conditions of a given ensemble change. However, the approximations which have to be taken for scanning full configurational space are not trivial and may lead to false predictions. Nonetheless, many artificial or hitherto unknown arrangements can be tested for kinetical survival with a remarkable accuracy.

Still, "science in mind" does not work in configurational space and scientists mostly rely on plausibility based on what they already know. In this respect, it is important to have guidelines along which imagination can extent. The concept of PSP is a deeply structural one, unifying symmetry and topology. It allows for well ordered ways of extending structural knowledge into the more complex, into the unknown. One such approach was given recently where interference of differently complicated PNS was shown to be a useful tool for inventing new structures in a systematic way. The approach is fairly simple and basically a merger of a more fundamental PNS' say

$$
\sum_{h}\left|S\left(\mathbf{h}^{\prime}\right)\right| \cos \left[2 \pi\left(\mathbf{h}^{\prime} r\right)-\alpha\left(\mathbf{h}^{\prime}\right)\right]=0
$$

with a slightly more modulated PNS"

$$
\begin{aligned}
& \sum_{h}\left|S\left(\mathbf{h}^{\prime}\right)\right| \cos \left[2 \pi\left(\mathbf{h}^{\prime} r\right)-\alpha\left(\mathbf{h}^{\prime}\right)\right] \\
& +\left|S\left(\mathbf{h}^{\prime \prime}\right)\right| \cos \left[2 \pi\left(\mathbf{h}^{\prime \prime} \mathbf{r}\right)-\alpha\left(\mathbf{h}^{\prime \prime}\right)\right]=0,
\end{aligned}
$$

where $\left|S\left(\mathbf{h}^{\prime \prime}\right)\right|$ is about $10 \%$ of $\left|S\left(\mathbf{h}^{\prime}\right)\right|$ and $\left|\mathbf{h}^{\prime \prime}\right|>\left|\mathbf{h}^{\prime}\right|$.
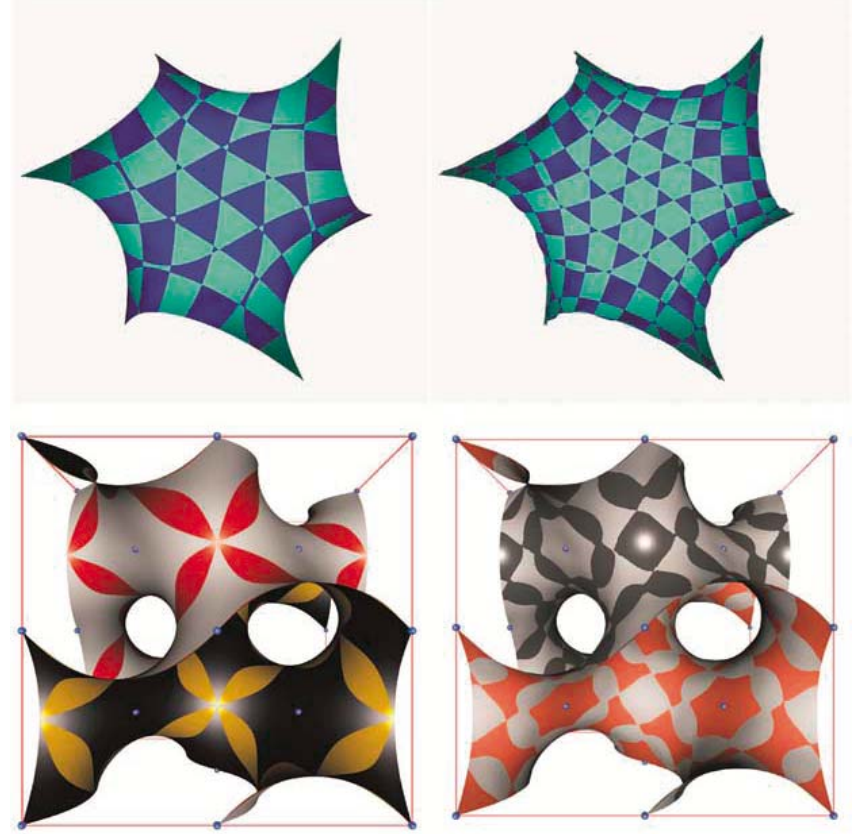

Fig. 10. Four tilings on the $G$ surface as PNS'. It is easy to construct nets from these patterns and cast them into hypothetical chemical frameworks.
As a PNS" by definition exhibits more structural details it has modulations which pop out PNS'. This generates systematic patterns or tilings on PNS' which are apt for interpretation (Fig. 10).

\section{Phase transitions - structural development in time}

The achievements in connecting periodic hyperbolic surfaces to the chemical structure of matter were soon followed by the idea of using this novel kind of structural description to elucidate mechanisms of reconstructive phase transitions.

In contrast to $2^{\text {nd }}$ order phase transitions, $1^{\text {st }}$ order reconstructive phase transitions involve generally larger scale rearrangements and are mechanistically much more demanding because there is no strict symmetry control as for the former. For $2^{\text {nd }}$ order transitions a distinct group-subgroup relation is a prerequisite, such that the transition proceeds according to one and only one irreducible representation of the subgroup. The symmetrical relations between structures connected through $1^{\text {st }}$ order transitions are more distant, and allow for a large variety of displacements of atoms or building blocks required to achieve the actual structural transition.

The application of PES to the investigation of reconstructive phase transitions is an attempt to replace the methodical complexity of having $\mathrm{n}$ particles in configurational space by an envelope surface to the structures in question. In that case PES are mostly more useful than PMS or PNS because they allow for a better adaptation of structure and surface. There is a huge number of transformation pathways to be considered possible at first sight, but most of them will not come into play either because they involve too much activation energy, or because the atomic displacements are too big to compete with a small number of transitions fulfilling both requirements: low activation barrier and short diffusive if not just only displacive movements of the basic particles. Starting from topological criteria puts the focus in the first instance not on energy considerations (and thus on thermodynamics) but on the magnitude of displacements (i.e. ultimately kinetics) and can therefore provide complementary information.

Whereas the advantage of this approach being computationally quite inexpensive is nowadays continually decreasing in importance, the origin of its computational economy remains of unvarying relevance: It is the fact that important information gathered in the diffraction patterns is being actually used instead of being discarded and replaced by blind computational power.

There are two principal alternatives in which periodic hyperbolic surfaces can be used in a phase transition study. Either the structure in question can be embedded in the surface such that all atoms are lying on the surface, or the surface can be chosen such as to envelope the structural network. The first approach has been applied to the austenite-martensite transformation by Hyde and Andersson [71-72], but the underlying transformation of the gyroide surface is a Bonnet-type transformation and as such very specific. The results cannot be generalized to systems where connectivity changes during the transition, i.e. to recon- 
structive phase transitions. Therefore the second approach seems more promising for the investigation of $1^{\text {st }}$ order transformations.

First attempts date back to early POPS work with H.-G. von Schnering in the 1980ies. He knew that the sodium chloride and the cesium chloride types are transformable into each other just by a change of the $c / a$ ratio from 1.22 to 2.44 in the trigonal setting. Consequently that transition was modeled by - at that time - quite crude and laborious graphical methods. Even though the lowest energy trajectory proceeds through another channel (no. 12 in Table 3) we were quite enthusiastic to find a concerted movement of all atoms from one structure to the other. Considering that one could guess the size of the volume which should undergo the transformation it was clear that the model could refer to everything between a tiny nucleus and a macroscopic volume, respectively. The nucleation, though is much more likely, because of the excitation of only a few atoms at a time. The first attempt to apply the older POPS approach of von Schnering and Nesper to the $\mathrm{NaCl}$-to-CsCl type transition was undertaken by Oehme [73]. The more general concept of PES as enveloping functions was first used by Leoni et al. in order to tackle reconstructive phase transitions [74] in the silica phase diagram, between carbon allotropes, and between several disilicide structure types.

One of the first actual examples was the study of the quartz-to-tridymite transition in the silica phase system, where PESs are used to envelope the structural networks of starting and final phases [74]. Linear interpolation between the two enveloping surfaces according to

$$
f(\text { transition })=v \cdot f(\text { phase } A)+(1-v) \cdot f(\text { phase } B),
$$

where

$$
v=[0 \ldots 1]
$$

leads to a gradual transformation from starting to final surface. Changes in the surface genus are interpreted as changes in network connectivity (i.e. in bonding), thus the evolution of one surface into the other reflects the changes in network geometry and topology of the two underlying structures for any given point on the transformation coordinate. In case of the quartz-to-tridymite transition, inspection of the intermediate surface leads to the proposal of an intermediate structure related to the $\alpha-\mathrm{B}_{2} \mathrm{O}_{3}$ and the $\alpha-\mathrm{ThSi}_{2}$ structure types. However, the transition model does not unambiguously account for the oxygen migration paths since at surface ruptures (network cleavages) the presumed oxygen location inside the $\mathrm{Si}-\mathrm{Si}$ connection tunnels can of course not be maintained in such cases. However, the trace of the Si movements allow to select oxygen candidates from the closer silicon environment to estimate the transition geometries and mutual oxygen movements.

Consecutively, other systems were investigated in a similar manner. The transitions between the cubic $\mathrm{SrSi}_{2}$ and the $\alpha-\mathrm{ThSi}_{2}$ type and their relations to the diamond structure were closely examined [75]. Moreover, the importance of a suitable choice of corresponding cell settings for the limiting structures is pointed out by means of a specific example.

Modeling of reconstructive phase transitions via PES is continued, and deepened with respect to reciprocal space considerations [76]. In the well-known B1-B2 transition several mechanistic propositions have been followed and compared.

Despite the abovementioned difficulties we were able to propose a new transition pathway for the quartz-totridymite transformation with a 5-coordinate transition state at two neighboring $\mathrm{Si}$ centers including oxygen displacements. A surprising result was worked out for the transformation between cubic $\mathrm{SrSi}_{2}$ and $\alpha-\mathrm{ThSi}_{2}$. The whole three-dimensional $\left[\mathrm{Si}^{-}\right.$] framework collapsed to acetyleneanalogue $\mathrm{Si}_{2}^{2-}$ units which after a rotation of dumb-bells in space, reconnect to the other $3 \mathrm{D}$ framework $\left[\mathrm{Si}^{-}\right]$. Another striking result was obtained for the switch from hexagonal graphite-to-hexagonal diamond (lonsdaleite). The lonsda-

\begin{tabular}{|c|c|c|c|c|}
\hline 1 & Transition & Space groups & Underlying concept & Literature \\
\hline 2 & $\mathrm{~B} 1-\mathrm{B} 2(\mathrm{NaCl}-\mathrm{CsCl})$ & $F m \overline{3} m-P m \overline{3} m$ & POPS & [77] \\
\hline 3 & Quartz - Tridymite & $P 6_{4} 22\left(P 6_{2} 22\right)-P 6_{3} / m m c$ & PES & {$[73]$} \\
\hline 4 & Tridymite - Cristobalite & $P 6_{3} / m m c-F d \overline{3} m$ & PES & {$[45]$} \\
\hline 5 & Cubic $\mathrm{SrSi}_{2}-\alpha-\mathrm{ThSi}_{2}$ & $F d \overline{3} m-I 4_{1} / a m d$ & PES, 2 pathways & [74] \\
\hline 6 & Cubic Diamond - rhombohedral Graphite & $F d \overline{3} m-R \overline{3} m$ & PES & \\
\hline 7 & Hex. Graphite - hex. Diamond (Lonsdaleite) & $P 6_{3} / m m c-P 6_{3} / m m c$ & PES & \\
\hline 8 & Cubic Diamond - hex. Graphite & $F d \overline{3} m-P 6_{3} / m m c$ & PES, 2 pathways & \\
\hline 9 & $\mathrm{~B} 1-\mathrm{B} 2(\mathrm{NaCl}-\mathrm{CsCl})$ & $F m \overline{3} m-P m \overline{3} m$ & PNS & \\
\hline 10 & $\alpha-\mathrm{Sn}-\beta-\mathrm{Sn}$ & $F d \overline{3} m-I 4_{1} / a m d$ & PES & {$[75]$} \\
\hline 11 & $\begin{array}{l}\text { Tin Dioxide } \\
\mathrm{CaCl}_{2} \text { type }-\alpha-\mathrm{PbO}_{2} \text { type } \\
\alpha-\mathrm{PbO}_{2} \text { type }-\mathrm{PdF}_{2} \text { type } \\
\mathrm{CaCl}_{2} \text { type }-\mathrm{PdF}_{2} \text { type }\end{array}$ & $\begin{array}{l}\text { Pnnm }-P b c n \\
P b c n-P a \overline{3} \\
\text { Pnnm }-P a \overline{3}\end{array}$ & PES & {$[75]$} \\
\hline 12 & $\mathrm{~B} 1-\mathrm{B} 2(\mathrm{NaCl}-\mathrm{CsCl})$ & $F m \overline{3} m-P m \overline{3} m$ & PES, 3 Pathways & {$[75]$} \\
\hline 13 & $\beta$-Quartz - Keatite & $P 6_{4} 22\left(P 6_{2} 22\right)-P 4_{3} 2_{1} 2$ & PES & [76] \\
\hline 14 & $\alpha-\mathrm{Eu}_{8} \mathrm{Ga}_{16-x} \mathrm{Ge}_{30+x}-\beta-\mathrm{Eu}_{8} \mathrm{Ga}_{16-x} \mathrm{Ge}_{30+x}$ & $P m \overline{3} m-I \overline{4} 3 m$ & PNS & {$[54]$} \\
\hline 15 & $\alpha-\mathrm{BaAl}_{2} \mathrm{Ge}_{2}-\beta-\mathrm{BaAl}_{2} \mathrm{Ge}_{2}$ & Pnma - I4/mmm & PNS & {$[56]$} \\
\hline
\end{tabular}

Table 3. Phase transitions analyzed by means of PMS or PNS. 
leite form is preferably found at meteorite craters hinting at a shockwave transition. Comparing the two tunnel systems embedding the lonsdaleite framework and that of the other PES side, respectively, reveals that the "empty side" has the topology of hexagonal graphite. The opposite is true for the hexagonal graphite structure. In other words the carbon atoms of hexagonal graphite just have to pass over into the void space and thus form the denser lonsdaleite.

Using the examples of the transformation of $\beta$-quartzto-keatite as well as several transitions in the tin dioxide phase system, a novel way of deducing common unit cells through suitable mutual orientation by analysis of the most intense reflections of the limiting structures in question was developed. Lately, important progress has been made on the problem of selecting common unit cells for the two limiting structures. This is subject to two partial problems: (i) Determination of matrices for the mutual orientational transformation of the unit cells; (ii) Choice of the optimal size of the supercell. Both can be derived from following reciprocal space considerations. (i) Most prominent structure factors with smallest $\mathbf{h}_{n}$ vectors (largest $d$-values) are rotated to map best onto each other. This yields the inverse rotation matrix for optimal real space mapping. (ii) The size of the $\mathbf{h}_{n}$ vector sphere which allows for the best structure factor mapping determines the size of the super structure [76]. The argument for mapping most prominent structure facture sets is, of course, keeping most prominent atomic planes intact or leave them as little changed as possible. The quartz-to-keatite transition was published in the last issue of this journal [77].

The intermetallic clathrate $\mathrm{Eu}_{8} \mathrm{Ga}_{16-x} \mathrm{Ge}_{30+x}$ undergoes a structural transition in dependence on temperature and composition. The crystal structure of the high-temperature $\beta$-phase corresponds to clathrate I type (space group $P m \overline{3} n$, Pearson symbol $c P 54)$, that of the low-temperature $\alpha$-phase is of clathrate VIII type (space group $I \overline{4} 3 m, c I 54)$. The organization of both structures is described with a PNS (Fig. 6). The structural relationship between the clathrate I and clathrate VIII structural patterns is successfully described with periodic minimal surfaces approximants. The description of the structural transition is formulated in terms of a linear interpolation between the two enveloping surfaces shown in Fig. 6 and visualizes a global transformation of the two four-connected covalent frameworks formed by $\mathrm{Ga}$ and Ge. Even the critical phenomena, like the local break of few bonds in the starting arrangement, the existence of three-connected atoms in the intermediate structural arrangements, and the formation of new bonds in the final arrangement in order to restore the four-connected pattern, are sufficiently reflected by the topology of the PNS used for the description [54].

A similar situation was observed between the modifications of the intermetallic phase $\mathrm{BaAl}_{2} \mathrm{Ge}_{2}$ [56]. The reversible, reconstructive phase transition between the lowtemperature $\alpha$-phase (space group Pnma, $\alpha$ - $\mathrm{BaCu}_{2} \mathrm{~S}_{2}$ type) and the high-temperature $\beta$-phase (space group $14 / \mathrm{mmm}$, $\mathrm{BaZn}_{2} \mathrm{P}_{2}$ type) is modeled with the help of PNS (cf. Fig. 6). A change from the layered $\beta$-phase to the 3D network of the $\alpha$-phase upon an increase of the puckering of the layers and the appearance of new bonds between them is well visualized by the observed topological features of the PNS during the transformation.

Assuming a concerted route of the structural transformation, the description of the transformation mechanism by means of continuous change of the enveloping periodic surface offers clear transparent views on the local changes of the crystal structure during the transformation. Further investigations of the structural transformations in particular in halides of the alkaline metals allying molecular dynamics in combination with the sampling approach revealed that these are proceeding via formation of the low-dimensional nucleation centers and are not following the concerted route. But even in this case, the PNSderived pathways deliver reasonable starting situations and allow to find the final configuration more efficiently [78-81].

\section{Applications to soft matter problems}

In soft matter research PMS are widely being applied to understand the creation and transformations of self-organized structures. This concerns liquid crystals, tenside solutions, amphiphile dispersions, and other related systems. Formation of micelles goes already beyond simple nucleation and may be understood as the start of a phase separation. Self-organized isolated or continuous compartments as vesicular double-membrane structures are dominated by surface tension. So it is not surprising that they tend to follow minimal surface requirements [82]. Corresponding phase systems have been derived and it is interesting to note that on increase of concentration of amphiphile molecules in solution only after passing a wide range of isolated compartmentation, induction of bicontinuous structures like hexagonal or cubic phases turn up (Fig. 11). In case of upcoming structural order a few Bragg reflections may be observed which allow to trace back the general PMS arrangement [83-85]. However, they are broad and only a few of the smallest $\mathbf{h}_{n}$ vectors.

Besides surface tension, mean and Gaussian curvatures are important measures for the energy of bicontinuous arrangements as well as for the classification of periodic hyperbolic surfaces which was worked out especially by Helfrich and coworkers [86]. The classification of curvatures is not an all in all easy process and several different approaches have been applied. We did inspect the counting box method which is used in the approximation of fractal behavior. The concept of fractal dimension is intrinsically related with the self-similarity property. For natural objects this can only be evaluated at final resolution thus they cannot display "ideal" fractal behavior. For obvious reasons - at increasing resolution there will always a limiting resolution of a natural object - practically all $\mathrm{Ri}$ chardson-Mandelbrot log-log curves based on real objects or processes appear concave. At low resolution a log-log curve might appear to be straight but a concave curve always shows up if the resolution is increased. Rigaut [87] derived a new mathematical model for determining asymptotic fractals which offers advantages compared with previous definitions [88]. Chermant and Bignon [89-91] were the first to note that the contours of most natural objects 


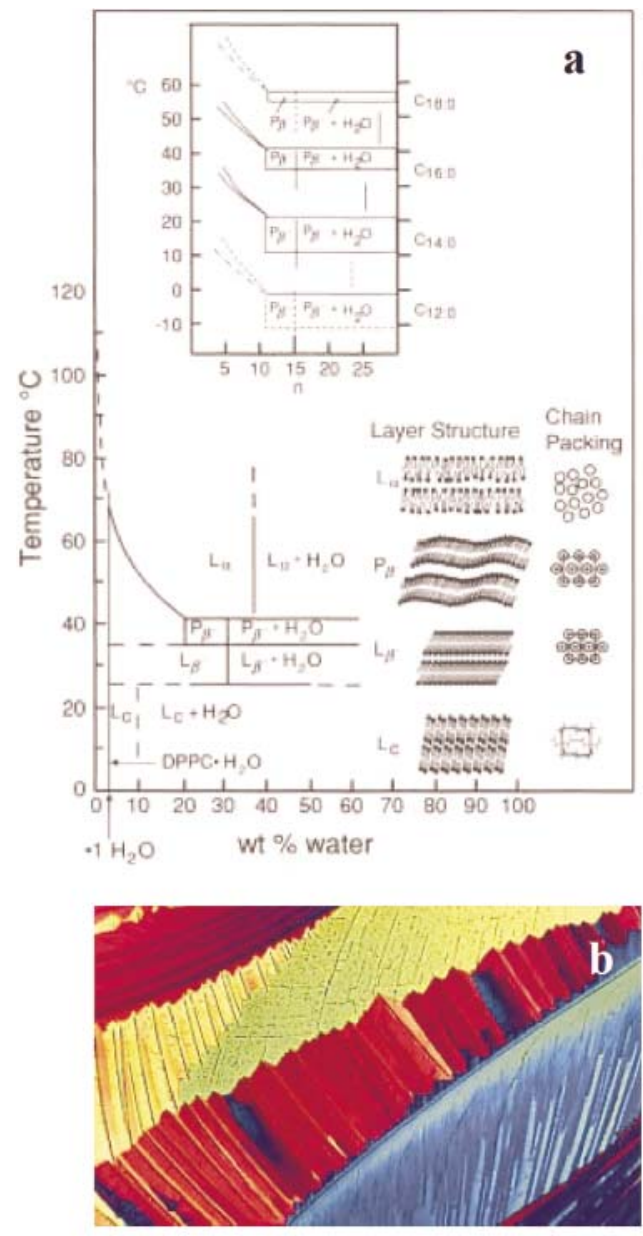

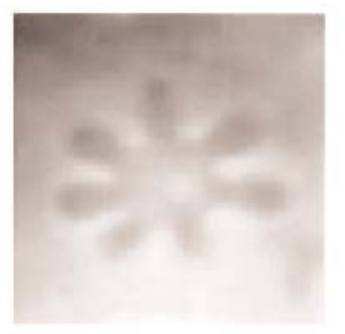
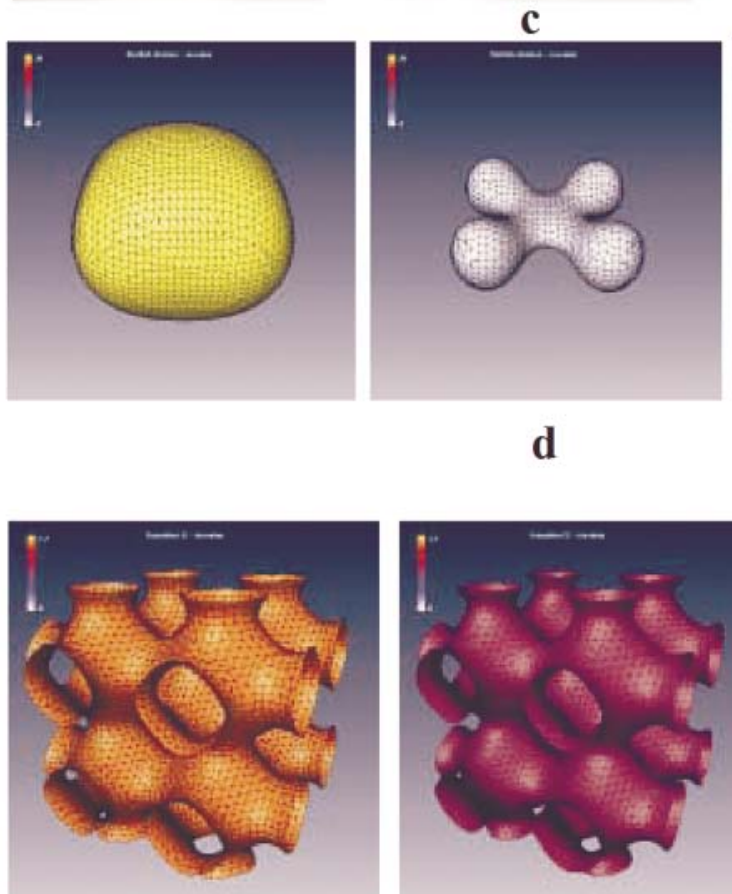

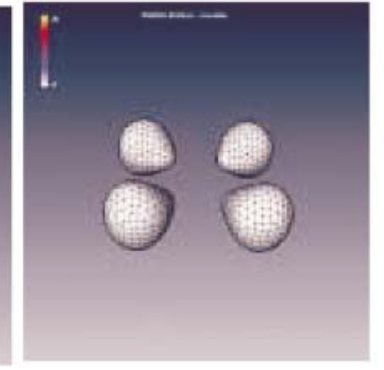

d
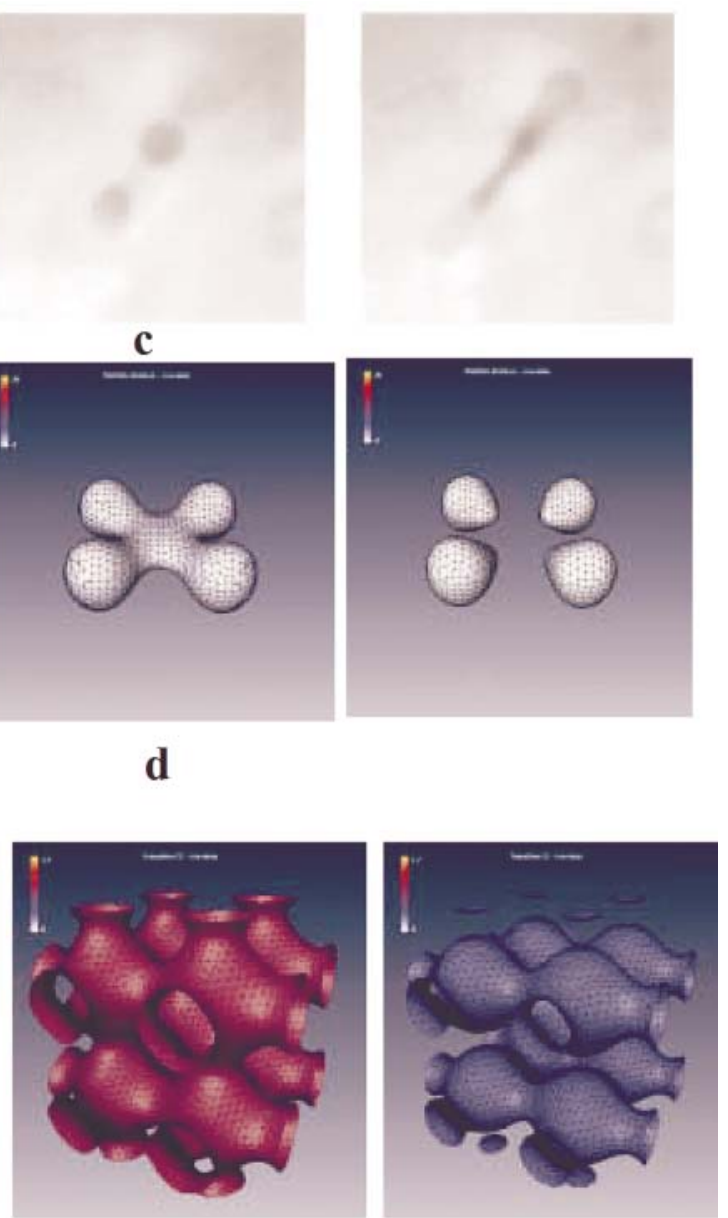

e

Fig. 11. (a) Phase diagram of egg lecithin, (b) smectic phase of oleic acid under polarized light; (c) starfish vesicles under the light microscope, (d) simulation of the self-separation of a spherical vesicle to a starfisch and finally to four independent vesicles, (e) simulation of a one dimensional separation of a bicontinuous $P$ surface to a 2 D layered variant [94].

show concave Richardson-Mandelbrot log-log plots, instead of linear ones (ideal fractality). Classic fractality is the ideal realization of a more general concept [92]. Rigaut [93] has conjectured that most linear RichardsonMandelbrot log-log plots that have been published were taken from objects explored at low resolution.

We did apply the box counting method to selected surface examples to check the numerical results (Fig. 12). Most of the structures are not self-similar at all, but some others can exhibit a certain degree of self-similarity. Anyway the evaluation of $D$ (see below) is a useful tool to evaluate the change in complexity during a transformation. The box-counting procedure has been applied at a resolution low enough to detect the difference in curvature between different objects [94]. In Fig. 13 an example of the method is shown applied on two 2-dimensional functions. The number of boxes is regulated by a power law of the form

$$
N_{D}=N_{E}^{D}
$$

where $N_{D}$ is the number of colored boxes. In order to evaluate the coefficient $D$, one can start from the following functions:

$$
y_{3}=N_{E}^{3}
$$

is the number of boxes in the raster and

$$
y_{D}=N_{E}^{D}
$$

is the number of boxes in the raster touching the function. The ratio is:

$$
\frac{y_{3}}{y_{D}}=\frac{N_{E}^{3}}{N_{E}^{D}}=N_{E}^{3-D},
$$

consequently

$$
\log \frac{y_{3}}{y_{D}}=(3-D) \log N_{E}
$$

and

$$
D=3-\log \frac{y_{3}}{y_{D}} \cdot \frac{1}{\log N_{E}}
$$

Let us consider three well known surfaces - cylinder, $G$ and $P$ surfaces. Their equations are shown in Table 4 . None of them is self-similar so they are not fractals. Nevertheless, defining some convenient resolutions, the box-counting procedure can be applied. Following the suggestion of Rigaut [92], one can limit the resolution to a range in which the substantial differences in terms of curvatures are captured. It is interesting to note that the $D$ value is fairly 

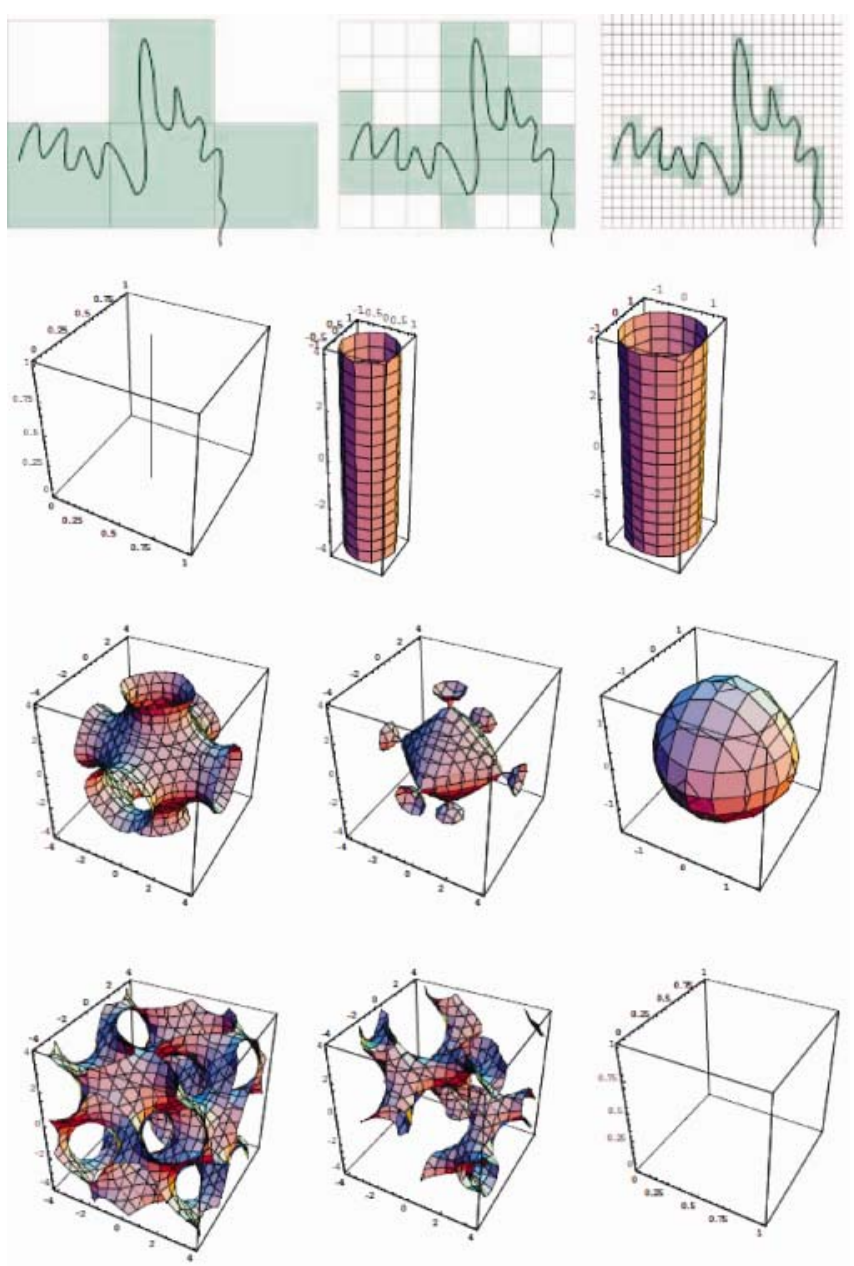

Fig. 12. (top) Schematic representation of the box counting method in a $1 \mathrm{D}$ case; (bottom) - $2 \mathrm{D}$ surfaces for $C=0,1$, and 2 , cylinder, $P$ surface, and Gyroid surface.

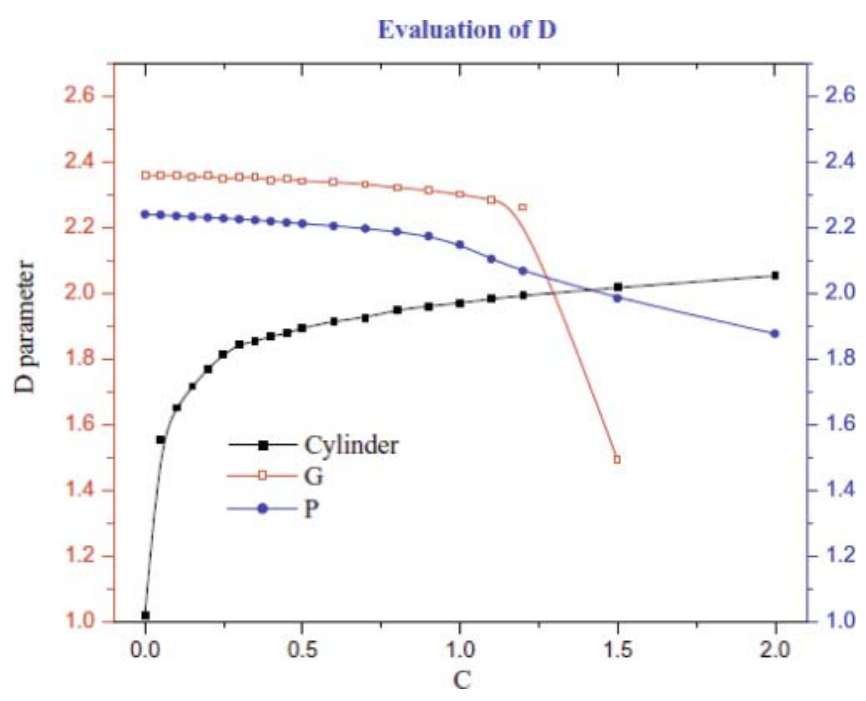

Fig. 13. Dependence of the value of $D$ on the parameter $C$ for cylinder, $P$ and $G$ surfaces.

Table 4. Analytical expressions for the surfaces Cylinder, $G$, and $P$.

\begin{tabular}{ll}
\hline Surface & Expression \\
\hline Cylinder & $x^{2}+y^{2}-C=0$ \\
$G$ & $\cos y \sin x+\cos z \sin y+\cos x \sin z-C=0$ \\
$P$ & $\cos x+\cos y+\cos z-C=0$ \\
\hline
\end{tabular}

Table 5. Parameter $D$ for the functions of Table 4 performed in a raster of size $=4 \mathrm{~h}$ ranging between 40 and 160 and error $\delta$.

\begin{tabular}{|c|c|c|c|c|}
\hline $\mathrm{C}$ & $G$ surface & $P$ surface & Cylinder & $\delta$ \\
\hline 0 & 2.359 & 2.240 & 1.021 & 0.04 \\
\hline 0.05 & 2.357 & 2.238 & 1.554 & 0.04 \\
\hline 0.1 & 2.360 & 2.235 & 1.652 & 0.04 \\
\hline 0.15 & 2.352 & 2.233 & 1.716 & 0.04 \\
\hline 0.2 & 2.357 & 2.230 & 1.770 & 0.04 \\
\hline 0.25 & 2.347 & 2.228 & 1.815 & 0.04 \\
\hline 0.3 & 2.353 & 2.226 & 1.844 & 0.04 \\
\hline 0.35 & 2.353 & 2.223 & 1.854 & 0.04 \\
\hline 0.4 & 2.343 & 2.219 & 1.869 & 0.04 \\
\hline 0.45 & 2.348 & 2.215 & 1.879 & 0.04 \\
\hline 0.5 & 2.341 & 2.212 & 1.895 & 0.04 \\
\hline 0.6 & 2.338 & 2.205 & 1.915 & 0.04 \\
\hline 0.7 & 2.332 & 2.197 & 1.926 & 0.04 \\
\hline 0.8 & 2.322 & 2.187 & 1.951 & 0.04 \\
\hline 0.9 & 2.313 & 2.173 & 1.961 & 0.04 \\
\hline 1 & 2.301 & 2.148 & 1.970 & 0.04 \\
\hline 1.1 & 2.285 & 2.105 & 1.983 & 0.04 \\
\hline 1.2 & 2.262 & 2.068 & 1.993 & 0.04 \\
\hline 1.5 & 1.494 & 1.985 & 2.018 & 0.04 \\
\hline 2 & 2 & 1.877 & 2.053 & 0.04 \\
\hline
\end{tabular}

stable for certain ranges of $C$ parameters. When it starts to change considerably, it seemingly has to do with the relation of object and box sizes (cf. Table 5). The graphical representation of this development is shown in Fig. 13.

\section{Outlook}

Topological analysis is a naturally preferred strategy of humans as predominantly visual creatures. The generality of periodic topological forms, especially of periodic surfaces can only be presaged until now. Such forms appear on different scales in completely different connections to matter and dynamics and generate a stunning intensity of attraction. They even have shown up as one-dimensional models for elementary particles, so-called skyrmions in which topological genus and charge are unified [95-98]. It has also been proposed that skyrmions may be useful models for describing magnetic metals [99]. Templated synthesis and self-organization in soft matter constitute enabling expertise for future nano materials and nano device research. It is still not finally clear to what extent plane wave decomposition of band structures and PSP topology can be correlated. This may also be true for lattice dynamics considerations. Although it may appear that choosing PSP approaches for understanding chemical and physical problems is discretionary to the author however we strongly believe that Paul Dirac statement applies: "This result is too beautiful to be false; it is more important to have beauty in one's equations than to have them fit experiment" [100]. Finally it should be mentioned that the existence of emergent properties is highly discussed in many fields from theoretical physics to neuroscience and 
brain-mind research [101-104]. Emergent states by definition would have to be ensemble states and such could find representations only by models of the ensemble as a whole.

Acknowledgments. Dr. A. Ormeci is acknowledged for Fermi surface calculations and Dr. B Bieri-Gross for assembling data on phase transition investigations. Thanks to A. Zürn for providing some pictures.

\section{References}

[1] R. Nesper, Von der Ästhetik als Modellierung der Gefühle und von den Emotionen als Grundlage unseren Wissens, Ed. Collegium Helveticum, Zürich 2009.

[2] W. Pauli, A. Hermann, K. von Meyenn, Victor, F. Weisskopf (Eds.), Scientific Correspondence with Bohr, Einstein, Heisenberg, a.o./Volume I-IV, Springer Berlin, Heidelberg, New York 1979-2005.

[3] J. L. Lagrange, Lagrange A Euler 1760, http://www.math.dartmouth.edu/ euler/correspondence/letters/ OO1367.pdf.

[4] H. A. Schwarz, Gesammelte Mathematische Abhandlungen, Vols 1, 2, Springer, Berlin 1890.

[5] D. Hilbert, S. Cohn-Vossen, Anschauliche Geometrie, Julius Springer Verlag, Berlin 1932.

[6] T. Radó, On Plateau's problem, Ann. of Math. 1930, 31, 457.

[7] A. H. Schoen, NASA Tech. Note D-5541; 1970; Not. Am. Math. Soc. 1969, 16, 19; 1968, 15, 929; 1968, 15, 727; 1967, 14, 661.

[8] C. Roland, Frei Otto - Spannweiten, Verlag Ullstein GmbH, Frankfurt 1965; The Museum of Modern Art: the work of Frei Otto, The Museum of Modern Art, New York 1972; J. J. Sweeney und J. L. Sert: Antoni Gaudí, Verlag Hatje, Stuttgart 1960.

[9] M. Burt, Spacial arrangement and Polyhedra with curved surfaces and their architectural applications, Dissertation, Technion Haifa, 1964; http://www.professormichaelburt.com/.

[10] M. Burt, Wachmann, Kleinmann, Infinite Polyhedra, Technion Publication, Haifa 1974.

[11] M. Burt, http://www.professormichaelburt.com.

[12] F. Otto, Tanzbrunnen Köln, Part of Rheinpark. Year of Construction: 1957. Architect: Josef Op Gen Oorth; Roof: Frei Otto. Back right: the Staatenhaus, date: 20. Mai 2010, photograph: Raimond Spekking.

[13] P. Pearce, Structure in Nature is a Strategy for Design, T. M. Press (Ed). The MIT Press, Cambridge, Massachusetts and London 1978.

[14] S. Andersson, On the description of complex inorganic crystal structures, Angew. Chem. Int. Ed. Engl., 1983, 22, 69-81; S. Andersson, S. T. Hyde, H. G. von Schnering, The intrinsic curvature of solids, Z. Kristallogr. 1984, 168, 1-17; S. Andersson, L. J. Fälth, An alternative description of the paulingite structure J. Solid State Chem. 1983, 46, 265-268.

[15] Ch. Baerlocher, L. B. McCusker, D. H. Olson, Atlas of Zeolite Framework Types, $6^{\text {th }}$ Ed. Elsevier Amsterdam 2007.

[16] C. Hermann, International Tables for the Determination of Crystal Structures, First Volume: Tables on the theory of groups, $1^{\text {st }}$ Ed., C. Hermann (Ed), McGraw-Hill, New York 1935; C. Hermann. Zur Nomenklatur der Gitterkomplexe. Z. Kristallogr. 1960, 113, 142-154.

[17] W. Fischer, E. Koch. Eine Definition des Begriffs „Gitterkomplex“. Z. Kristallogr. 1974 139, 268-278.

[18] W. Fischer, E. Koch; International Tables for Crystallography A, 3rd Ed., Th. Hahn (Ed.), Kluwer Dordrecht 1992, 826-854.

[19] L. M. Gelato, E. Parthe, Structure TIDY - a computer-program to standardize crystal-structure data, J. Appl. Cryst. 1987, 20, 139-143.

[20] P. Niggli, Topological structure analysis II, Z. Kristallogr. 1928, 68, 404-466.

[21] P. G. L. Dirichlet, Crelle's Journal 1850, 40, 209; In: Dirichlet's Werke in zwei Bänden, Bd. 1, L. Kronecker (Hrsg.), Georg Reimer, Berlin 1889; Band 2, L. Kronecker, L. Fuchs (Hrsg.), Georg Reimer, Berlin 1897.

[22] G. F. Voronoi, Crelle's Journal 1908, 98, 134, 138; In: Gesammelte Werke in drei Bänden (russ.). I. M. Winogradow (Hrsg.),
Verlag der Akademie der Wiss. der Ukr. SSR, Kiew 1952/5; R. Klein, Algorithmische Geometrie, Springer 2005.

[23] E. Hellner, E. Koch, A. Reinhardt, The homogeneous frameworks of the cubic crystal structures. - Physik Daten - Physics Data 1981, 16-2, 1-67.

[24] H. Sowa, E. Koch, W. Fischer, Orthorhombic sphere packings. II. Bivariant lattice complexes, Acta Crystallogr. 2007, A63, 354-364; H. Sowa, W. Fischer, Orthorhombic sphere packings. I. Invariant and univariant lattice complexes, Acta Crystallogr. 2006, A62, 413-418; H. Sowa, E. Koch, Hexagonal and trigonal sphere packings. IV. Trivariant lattice complexes of trigonal space groups, Acta Crystallogr. 2006, A62, 379-399; T. E. Dorozinski, W. Fischer, A novel series of sphere packings with arbitrarily low density, Z. Kristallogr. 2006, 221, 563-566; E. Koch, W. Fischer \& H. Sowa, Interpenetration of homogeneous sphere packings and of two-periodic layers of spheres, Acta Crystallogr. 2006, A62, 152-167; W. Fischer, Tetragonal sphere packings: minimal densities and subunits, Acta Crystallogr. 2005, A61, 435-444; W. Fischer, Minimal densities of cubic sphere-packing types, Acta Crystallogr. 2004, A60, 246-249; H. Sowa, E. Koch, Hexagonal and trigonal sphere packings. II. Bivariant lattice complexes, Acta Crystallogr. 2004, A60, 158-166; H. Sowa, E. Koch, W. Fischer, Hexagonal and trigonal sphere packings. I. Invariant and univariant lattice complexes, Acta Crystallogr. 2003, A59, 317326; W. Fischer, E. Koch, Homogeneous sphere packings with triclinic symmetry, Acta Crystallogr. 2002, A58, 509-513; E. Koch, W. Fischer, Sphere packings and packings of ellipsoids. In: International Tables for Crystallography, Vol. C, Second revised Edition, Kluwer Academic Publishers, Dordrecht, Boston, London 1999, 738-743; W. Fischer, Tetragonal sphere packings. III. Lattice complexes with three degrees of freedom, Z. Kristallogr. 1993, 205, 9-26.

[25] A. F. Wells, Structural Inorganic Chemistry, $5^{\text {th }}$ Ed., Oxford Science Publications, Clarendon 1984.

[26] W. B. Pearson, The Crystal Chemistry and Physics of Metals and Alloys, Wiley 1972.

[27] R. Nesper, H. G. von Schnering, Periodic potential surfaces in crystal structures, Angew. Chem. 1986, 98, 111-113.

[28] Yu. Grin, U. Wedig, F. R. Wagner, H. G. von Schnering, A. Savin, The Analysis of "Empty" Space in the PdGa5 Structure. $J$. Alloys Compd. 1997, 255, 203-208.

[29] H. G. von Schnering, R. Nesper, How nature adapts chemical structures to curved surfaces. Angew. Chem. Int. Ed. Engl. 1987, 26, 1059-1080.

[30] W. Fischer, E. Koch, On 3-periodic minimal surfaces, Z. Kristallogr. 1987, 179, 31-52.

[31] S. T. Hyde, http://epinet.anu.edu.au/mathematics/ minimal_surfaces.

[32] R. Holyst, Liquid crystals - Infinite networks of surfaces, Nature Materials 2005, 4, 510.

[33] F. Bertaut, Lenergie electrostatique de reseaux ioniques, J. Phys. Radium 1952, 13, 499.

[34] F. Bertaut, J., Electrostatic potentials, fields and field gradients, J. Phys. Chem. Solids 1978, 39, 97.

[35] H. G. von Schnering, Homonucleare Bindungen bei Hauptgruppenelementen, Angew. Chem. 1981, 93, 44; Angew. Chem. Int. Ed. Engl. 1981, 20, 33.

[36] H. G. von Schnering, R. Nesper, The curvature of chemical structures. Colloque de Physique 1990, C7, 383-396.

[37] H. G. von Schnering, R. Nesper, Nodal surfaces of Fourier series: fundamental invariants of structured matter, Z. Phys. B Condensed Matter, 1991, 83, 407-412.

[38] H. G. von Schnering, unpublished results 1993.

[39] S. Andersson, M. Jacob, S. Lidin, On the shape of the crystals, Z. Kristallogr. 1995, 210, 3-4.

[40] S. Andersson, M. Jacob, S. Lidin, Structure of the cubosome a closed lipid bilayer aggregate, Z. Kristallogr. 1995, 210, $315-318$.

[41] S. Andersson, M. Jacob, The mathematics of structures. The exponential scale, R. Oldenbourg Verlag, 1997.

[42] S. Andersson, S. Lidin, M. Jacob, The exponential scale and crystal structures, Z. Kristallogr. 1995, 210, 826-831.

[43] M. Jacob, S. Andersson, The nature of mathematics and the mathematics of nature, Elsevier, 1998. 
[44] P. J. F Gandy, J. Klinowski, Nodal surface approximation to the zero potential surfaces for cubic lattices, J. Math. Chem. 2002, 11, 1-16.

[45] P. J. F. Gandy, J. Klinowski, The equipotential surfaces of cubic lattices, Chem. Phys. Lett. 2002, 360, 541-551.

[46] A. Mackay, Periodic surfaces from finite element methods, Chem. Phys. Lett. 1994, 221, 317-321.

[47] U. Dierkes, S. Hildebrandt, A. Küster, O. Wohlrab, Minimal Surfaces, Springer-Verlag, 1991.

[48] M. Predel, Yu. Grin, Periodic Nodal Surfaces and Exponential Scale, Z. Kristallogr. 2011, 226, in press.

[49] S. Leoni, Applications of Periodic Surfaces for the Study of Crystal Structures, First Order Phase Transitions, Force Ordering, and Systematic Generation of Novel Structure Models, Dissertation, ETH no 12783, Zürich 1998.

[50] P. Villars, L. D. Calvert (Eds.), Pearson's Handbook of Crystallographic Data for Intermetallic Phases, ASM, $1^{\text {st }}$ Ed., Cleveland, $\mathrm{OH} 1985$.

[51] G. Bricogne, Methods and Programs for Direct-Space Exploitation of Geometric Redundancies, Acta Crystallogr. 1976, A32, 832-847.

[52] Y. Schumacher, Investigations of Highly Reactive Molecules in Zeolithe-Confined Materials, Dissertation, ETH no 11758, Zürich 1996; S. Brenner, L. B. McCusker, Ch. Baerlocher, Use a structure envelope to facilitate structure solution from powder diffraction data, J. Appl. Crystallogr. 1997, 30, 1167-1172.

[53] S. Brenner, L. B. McCusker, Ch. Baerlocher, The application of structure envelopes in structure determination from powder diffraction data, J. Appl. Crystallogr. 2002, 35, 243-252.

[54] L. G. Akselrud, Yu. Grin, unpublished results 2011.

[55] S. Leoni, W. Carrillo-Cabrera, Yu. Grin, Modeling of the clathrate VIII - clathrate I phase transition in $\mathrm{Eu}_{8} \mathrm{Ga}_{16} \mathrm{Ge}_{30}$, J. Alloys Comp. 2003, 350, 113-122.

[56] H. G. von Schnering, A. Zürn, J.-H. Chang, M. Baitinger, Yu. Grin, The intrinsic shape of the cubic $c P 124$ clathrate structure, Z. Anorg. Allg. Chem. 2007, 633, 1147-1153.

[57] S. Leoni, W. Carrillo-Cabrera, W. Schnelle, Yu. Grin, $\mathrm{BaAl}_{2} \mathrm{Ge}_{2}$ : synthesis, crystal structure, magnetic and electronic properties, chemical bonding, and atomistic model of the transition, Solid State Science 2003, 5, 139-148.

[58] M. Kohout, A Measure of Electron Localizability, Int. J. Quantum Chem. 2004, 97, 651-658.

[59] M. Kohout, F. R. Wagner, Yu. Grin, Atomic shells from the electron localizability in momentum space, J. Quant. Chem. 2006, 106, 1499-1507.

[60] M. Kohout, Bonding indicators from electron pair density functionals, Faraday Discuss. 2007, 135, 43-54.

[61] F. R. Wagner, V. Bezugly, M. Kohout, Yu. Grin, Charge decomposition analysis of the Electron Localizability Indicator: a bridge between the orbital and direct representation of the chemical bond, Chem. Eur. J. 2007, 13, 5724-5741.

[62] F. R. Wagner, M. Kohout, Yu. Grin, Direct Space Decomposition of ELI-D: Interplay of Charge Density and Pair-Volume Function for Different Bonding Situation, J. Phys. Chem. A 2008, 112, 9814-9828.

[63] Yu. Grin, U. Wedig, H. G. von Schnering, Hyperbolische Elektronenpaar-Struktur in $\mathrm{RhBi}_{4}$. Angew. Chem. 1995, 107, 13181320; Angew. Chem. Int. Ed. Engl. 1995, 34, 1204-1206.

[64] Yu. Grin, A personal opinion on Crystallography, Z. Kristallogr. 2002, 217, 311-312.

[65] Yu. Grin, F. R. Wagner, M. Armbrüster, M. Kohout, A. LeitheJasper, U. Schwarz, U. Wedig, H. G. von Schnering, $\mathrm{CuAl}_{2}$ revisited: Composition, crystal structure, chemical bonding, compressibility and Raman spectroscopy. J. Solid State Chem. 2006, 179, 1707-1719.

[66] Yu. Grin, K. Peters, H. G. von Schnering, Refinement of the Crystal Structure of Palladium Pentagallide, $\mathrm{PdGa}_{5}$. Z. Kristallogr. NCS 1997, 212, 6.

[67] R. Pöttgen, V. Hlukhyy, A. Baranov, Yu. Grin, Crystal structure and chemical bonding of $\mathrm{Mg}_{3} \mathrm{Ru}_{2}$. Inorg. Chem. 2008, 47, $6051-6055$.

[68] W. Carrillo-Cabrera, S. Budnyk, Yu. Prots, Yu. Grin, $\mathrm{Ba}_{8} \mathrm{Ge}_{43}$ revisited: a $2 a^{\prime} \times 2 a^{\prime} \times 2 a^{\prime}$ superstructure of the clathrate-I type with full vacancy ordering. Z. Anorg. Allg. Chem. 2004, 630, 2267-2276.
[69] V. Heine, D. Weaire, Pseudopotential theory of cohesion and structure, Solid State Phys. 1970, 24, 249; R. Nesper, Bonding patterns in intermetallic compounds, Angew. Chem. Int. Ed. Engl. 1991, 30, 789-817.

[70] H. Aoki, M. Koshino, D. Takeda, H. Morise, Electronic structure of periodic curved surfaces: Topological band structure, Phys. Rev. 2002, B65, 35102.

[71] S. T. Hyde, Ph.D. Thesis, Monash University 1986.

[72] S. T. Hyde, S. Andersson, K. Larsson, Differential geometry of a model membrane consisting of a lipid bilayer with a regular array of protein units. Z. Kristallogr. 1986, 174, 237.

[73] M. Oehme, Nullpotentialflächen und ihre Anwendung in der Kristallchemie, Dissertation, University of Stuttgart 1989.

[74] S. Leoni, R. Nesper, Elucidation of simple pathways for reconstructive phase transitions using periodic equi-surface (PES) descriptors. The silica phase system. I. Quartz-tridymite, Acta Crystallogr. A. 2000, 56, 383-393.

[75] S. Leoni, R. Nesper, Elucidation of simple pathways for reconstructive phase transitions using periodic equi-surfaces (PES) descriptors. II. The strontium disilicide transition, Solid State Sciences 2003, 5, 95-107.

[76] B. Bieri-Gross, Dissertation, ETH no. 16990, Zürich 2007.

[77] B. Bieri-Gross, R. Nesper, Topological modeling of reconstructive phase transitions through periodic hyperbolic surfaces: the $\beta$-quartz to keatite type transition. Z. Kristallogr. 2011, 226, in press.

[78] S. Leoni, D. Zahn, Putting the Squeeze on $\mathrm{NaCl}$ : Modelling and Simulation of the Pressure Driven B1-B2 Phase Transition, Z. Kristallogr. 2004, 219, 339-344.

[79] D. Zahn, S. Leoni. Mechanism of the Pressure-Induced Reconstructive Phase Transformation of $\mathrm{KCl}$ from the $\mathrm{NaCl}$ type to the $\mathrm{CsCl}$ type Structure. Z. Kristallogr. 2004, 219, 345-347; D. Zahn, S. Leoni, Nucleation and Growth in Pressure-Induced Phase Transitions from Molecular Dynamics Simulations: Mechanism of the Reconstructive Transformation of $\mathrm{NaCl}$ to the CsCl-type structure. Phys. Rev. Lett. 2004, 92, 250201-04.

[80] D. Zahn, Z. Kristallogr. 2011, 226, in press.

[81] S. Leoni, Z. Kristallogr. 2011, 226, in press.

[82] S. T. Hyde, Bicontinuous structures in lyotropic liquid crystals and crystalline hyperbolic surfaces, Current Opinions in Solid State \& Materials Science 1996, 1, 653-662.

[83] V. Luzzati, X-ray diffraction studies of lipid-water systems. In: Biological Membranes, D. Chapman (Ed.) Academic Press; 1968, 71-123.

[84] K. Larsson, Two cubic phases in monoolein-water system, Nature 1983, 304, 664.

[85] V. Luzzati, The micellar cubic phases of lipid-containing systems: analogies with foams relations with the infinite periodic minimal surfaces, sharpness of the polar-apolar partition, $J$. Phys. II (France) 1996, 6, 405-416.

[86] W. Helfrich, H. Rennschuh, Landau theory of the lamellar-tocubic phase transition. J. Phys. (France) Colloque 1990, 51, C7-169-190; W. Helfrich, Lyotropic lamellar phases. J. Phys. Cond. Mat. 1994, 6, A79.

[87] J. P. Rigaut, D. Schoeveaert-Brossault, A. M. Downs, G. Landini, Fractals in biology and medicine, F. Birkhauser (Ed.), Basel 1997.

[88] J. P. Rigaut, An empirical formulation relating boundary lengths to resolution in specimens showing non-ideally fractal dimensions, J. Microsc. 1984, 133, 41.

[89] J. L. Chermant, M. Coster, In: Proc. Int. Symp. Quant. Metallography, 1978, 125.

[90] M. Coster, A. Deschanvres, Prakt. Metall. Sp. Issue 1978, 8, 61.

[91] J. Bignon, Automatic measurement of alveolar surface of RAT LUNG using quantitative televisor microscope, Compt. Rend. Acad. Sci. Paris D. 1969, 269, 409.

[92] J. P. Rigaut, In: Biologie Theorique. E. Kretzschmar (Ed.), 1986, $141 \mathrm{ff}$.

[93] J. P. Rigaut, In : Fractals - Dimensions non entieres et application, D. Cherbit (Ed), Paris, 1987, $231 \mathrm{ff}$.

[94] S. Piotto Piotto, Dissertation, ETH no. 13593, Zürich 2000.

[95] J. K. Perring, T. H. R. Skyrme, A model unified field equation, Nucl. Phys. 1962, 31, 550-555.

[96] U. Enz, Die Dynamik der Blochschen Wand, Helv. Phys. Acta 1964, 37, 245-251. 
[97] A. C. Scott, Nonlinear Science: Emergence and Dynamics of Coherent Structures, $2^{\text {nd }}$ Ed. Oxford 2003.

[98] N. S. Manton, P. M. Sutcliff, Topological Solitons, Cambridge University Press 2004.

[99] U. K. Rössler, A. N. Bogdanov, C. Pfleiderer, Spontaneous skyrmion ground states in magnetic metals, Nature 2006, 442, 797-801.

[100] http://www.dirac.ch/PaulDirac.html.

[101] R. B. Laughlin, A Different Universe: Remaking Physics from the Bottom Down, Basic Books, New York, 2005.

[102] A. C. Scott, The Nonlinear Universe: Chaos, Emergence, Life, Springer 2010.

[103] H.-P. Dürr, Warum es ums Ganze geht, oekom Verlag, München 2009.
[104] H. Atmanspacher, H. Primas, Recasting Reality: Wolfgang Pauli's Philosophical Ideas and Contemporary Science, Springer 2009.

[105] B. Bitterli, Unpublished results.

[106] E. Koch, W. Fischer, Z. Kristallogr. 1996, 211, 251.

[107] R. Nesper, In: 20 Jahre Wilhelm-Klemm-Stiftung, pp. 137148, Shaker Verlag Aachen 2006.

[108] S. Wengert, R. Nesper, Localization Pattern in the Interstitial Space: a Special property of the Electron Localization Function, Chem. Eur. J. 1997, 3, 985.

[109] R. Nesper, S. Leoni, On Filings and Patterns on Hyperbolic Surfaces and Their Relation to Structural Chemistry, Chem. Phys. Chem. 2001, 2, 413. 